\title{
A new look at the derivation of the Schrödinger equation from Newtonian mechanics
}

\author{
L. Fritsche ${ }^{1, *}$ and M. Haugk ${ }^{2}$ \\ ${ }^{1}$ Institut für Theoretische Physik der Technischen Universität Clausthal, 38678 Clausthal-Zellerfeld, \\ Germany \\ ${ }^{2}$ HPC-Auto, Hewlett-Packard GmbH, 71065 Sindelfingen, Germany
}

Received 3 April 2003, revised 26 May 2003, accepted 28 May 2003 by F. W. Hehl

Published online 15 July 2003

Key words Schrödinger equation, stochastic quantum mechanics, vacuum fluctuations. PACS 02.50.Fz, 03.65.Ta, 05.40.-a

\begin{abstract}
We present a modified version of Nelson's seminal paper on the derivation of the time-dependent Schrödinger equation which draws on the equation of motion of a particle that moves under the influence of a classical force field and additional stochastic forces. The emphasis of our elaboration is focused on the implication of allowing stochastic forces to occur, viz. that the energy $E$ of the particle is no longer conserved on its trajectory in a conservative force field. We correlate this departure $\Delta E$ from its classical energy with the energy/time uncertainty relation $\Delta E \Delta t \approx \hbar / 2$ where $\Delta t$ is the average time for $\Delta E$ to persist. The stability of atoms, the zero-point energy of oscillators, the tunneling effect and the diffraction at slits are shown to be directly connected with the occurrence of such energy fluctuations. We discuss and rederive Nelson's theory entirely from this point of view and generalize his approach to systems of $N$ particles which interact via pair forces. Achieving reversibility in a description of particle motion that is akin to Brownian motion, represents a salient point of the derivation. We demonstrate that certain objections raised against Nelson's theory are without substance. We also try to put the particular worldview of this version of stochastic quantum mechanics into perspective with regard to the established Copenhagen interpretation.
\end{abstract}

\section{Introduction}

The conceptual foundation of modern quantum mechanics still rests on the idea that the motion of microscopic particles can consistently be described only if one accounts for the influence of the measuring process which leads to a completely new structure of the theory. The present study is concerned with adopting the opposite view in that it denies the fundamental role of the measuring process as being responsible for the non-classical behavior of microscopic particles. In spirit this is in keeping with John Bell's article "Against 'measurement' " [1]. We attempt to revitalize the idea of tracing the one-particle Schrödinger equation back to Newton's second law $\mathbf{F}=m_{0}$ a where $\mathbf{F}$ is the force acting on a particle of rest mass $m_{0}$ and effecting the acceleration a. As has been shown in the mid-60s by E. Nelson [2] a consistent derivation of the Schrödinger equation is, in fact, possible if one assumes that the particle in question is subject to a modified Brownian motion with a diffusion constant $\hbar / 2 m_{0}$ where $h=2 \pi \hbar$ is Planck's constant. Nelson's approach which is now commonly referred to as "stochastic quantization" has served ever since as a reference for numerous workers in the field of stochastic processes (see e.g. Gilson [3], Olavo [4], Blanchard et al. [5] and for earlier reviews by Yasue [6] and De La Peña [7]). Rather than discussing "stochastic quantization" primarily within the mathematical framework of stochastic processes we shall strongly emphasize the physical content of the assumptions made in going beyond classical mechanics. We shall stay as close to basic calculus as possible and try to make our exposition fully self-contained.

\footnotetext{
* Corresponding author E-mail: fritsche@pt.tu-clausthal.de
} 
The current analysis follows essentially Nelson's considerations but we depart from his presentation to make one aspect particularly evident: the reason why microscopic and macroscopic particles seem to move and to behave differently reflects the active role of the vacuum providing the space for energy fluctuations. The latter will henceforth be referred to as vacuum fluctuations. They have to be regarded as an objective property of nature. One could view them as caused by an exchange of energy between the mechanical system in question and the embedding vacuum that serves as an energy reservoir in terms of virtual particles: if that reservoir reduces its content of virtual particles, the energy of the system under study increases so that the energy of the entire system comprising this "vacuum reservoir" is conserved. In that sense quantum mechanical systems may be viewed as open systems like classical point mass systems in contact with a heat bath. This analogy will become particularly visible with our treatment. In the following we shall focus on the description of the point mass "subsystem" as an open system whose energy is conserved only on the average.

An implication of this concept is that charged point masses, despite their irregular motion, do not emit or absorb radiation, at least on the average. Radiation only occurs when the probability density of finding the point mass at its various positions in space becomes time-dependent.

If one disregards the details of the energy transfer between the two systems, vacuum fluctuations appear as an irregular temporary departure of the particle in question from its energy conserving trajectory in that it changes its energy by an average amount $\Delta E$ for an average time interval $\Delta t$ so that $\Delta E \Delta t \approx \hbar / 2$. It is this departure from classical energy conservation which explains the stability of the hydrogen atom in its ground-state (which applies quite generally to all atoms and their compounds), the zero-point motion of atoms in molecules and solids and the "tunneling" of particles through a potential wall which actually amounts to overcoming that wall. This process also governs the decay of radioactive nuclei and hence controls objectively the outcome of "Schrödinger's cat" experiment.

It also seems to be undebated that the material property of $\mathrm{He}^{4}$ to stay fluid at normal pressure down to the lowest accessible temperatures, is due to the zero-point motion of its atoms which keeps the system molten. Following the same line of thought, one can simply understand why it solidifies under pressure.

All these phenomena cannot possibly be caused by the interaction with observers or with measuring instruments.

The idea of viewing the motion of microscopic particles as a result of stochastic processes is much in the spirit of Einstein's early conjecture, that quantum mechanics takes “ $\ldots$ an approximately analogous position to the statistical mechanics within the framework of classical mechanics".

The interpretation of $\Delta E \Delta t \approx \hbar / 2$ as describing "out of mass shell"-phenomena that occur without observer has a long history. The short-range character of internuclear forces mediated by pions has commonly been explained by assuming that pions can spontaneously occur out of the vacuum by temporarily violating energy conservation. The amount of energy needed to create a pion having the rest mass of $140 \mathrm{MeV}$ and moving at half the velocity of light is $\approx 161 \mathrm{MeV}$. Its lifetime $\tau$ is defined by $\Delta E \tau \approx \hbar$ which yields $\tau=4 \cdot 10^{-24} \mathrm{~s}$. During that time it traverses a distance of $\approx 0.6 \cdot 10^{-13} \mathrm{~cm}$ which is consistent with experimental facts that bear on the range of internuclear forces. "Virtual particles" that occur "out of mass shell", that is for the same reason as the pions just discussed, constitute absolutely common objects in quantum field theory, like electron-positron pairs, and, more general, particle-anti-particle pairs.

Considering the epistemological attractiveness of tracing quantum mechanical behavior back to energy fluctuations in systems of point masses that are in a well defined sense open, it is hard to understand why the process of measurement is still defended as providing the fundamental rationale for the non-classical behavior of microscopic systems. We quote here only one voice (A. Zeilinger [8]) out of a more recent discussion on this matter: "I suggest that the very austerity of the Copenhagen interpretation, unsurpassed by that of any other interpretation of quantum mechanics, speaks very much in its favor. Indeed, its basic attitude toward the fundamental role of observation represents a major intellectual step forward over naive classical realism. In classical physics, observation is often regarded as a secondary concept, with the elements of the real world being primary. Yet it is obvious that any statement about nature has to be based on observation. 
What could then be more natural than a theory in which observation plays a more fundamental role than in a classical worldview?"

The particular situations discussed in the context of performing a measurement define in all cases a quantum mechanical system that undergoes changes in time objectively dictated by the time-dependent Schrödinger equation which will be shown to result from vacuum fluctuations. In the school of thought that believes in the crucial role of observations this fundamental equation falls essentially out of the blue. Moreover, there is no cogent interconnection between the influence of observation on a system and the mapping of its "observables" on Hermitian operators, which constitutes the standard procedure in setting up the mathematical framework of quantum mechanics. In a quantum mechanical theory that is based on vacuum fluctuations, the specific form of the operators and their commutation rules represent results of the theory and not assumptions. It is therefore hard to see that the Copenhagen interpretation effects "austerity" in the development of the mathematical apparatus.

The hypothesis of vacuum fluctuations allows one to view the "real world" in familiar terms of "naive realism". In an electron two-slit experiment, for example, this view suggests that each electron follows an irregular trajectory from the tip of the cathode to the fluorescent screen or some other position sensitive detector where it is captured by some atom. The latter process is described by the time-dependent Schrödinger equation whose Hamiltonian contains all the information on the interaction of the particles involved. If the electron is captured by an atom of a fluorescent screen the process is followed then by the ejection of a photon. One could position a digital camera behind that screen so that the photon, if it runs through the camera lens, could finally be monitored as a scintillation flash at a particular point of the camera display. In so doing, one could identify the position of the atom that captured the electron. However, the presence or absence of the camera behind the screen has no influence at all on the capturing process. It is hence absolutely unclear why that process should play a particular role as a measurement different from other electron capture processes which occur constantly in all kinds of situations and are governed by the same time-dependent Schrödinger equation. Heisenberg's statement [9] “... the idea of an objective real world whose smallest parts exist objectively in the same sense as stones or trees exist, independently of whether or not we observe them ... is impossible" and that "We can no longer speak of the behavior of the particle independently of the process of observation" [10] seems absurd in the light of the above considerations. But it reflects exactly the Copenhagen interpretation of quantum mechanics the spirit of which is still very much alive in practically all modern textbooks. The present state of affairs is perfectly described by Goldstein [11] in a more recent article on "Quantum Theory without Observers": "Many physicists pay lip service to the Copenhagen interpretation, and in particular to the notion that quantum mechanics is about observation or results of measurement. But hardly anybody truly believes this any more - and it is hard for me to believe that anyone really ever did." We refrain here from discussing the vast literature on this subject any further.

A number of post-war studies (cf. [12-15]) pursuing ideas similar to Nelson's and reflecting a certain mood of "post-war heresy" appeared prior to Nelson's paper along with other contributions that challenged the established interpretation of quantum mechanics.

For the sake of clarity in understanding the particular features of our approach, we deliberately omit discussing the literature that has grown out of Nelson's fundamental contributions [2,16,17]. A comprehensive review of this literature up to 1981 has been given by Selleri and Tarozzi [18]. More recent contributions of different scope are due to Bohm and Hiley [19] and to Olavo [4]. In Sect. 10 we shall only comment on one article that bears directly on the validity of the argument pursued in the current study.

As has already become evident from our considerations above, the existence of continuous, though irregular, particle trajectories will be a crucial hypothesis which all our studies in the ensuing sections will be based upon. Many of our discussions will therefore be in line with those familiar from de Broglie-BohmVigier theory [20-23, 25]. 


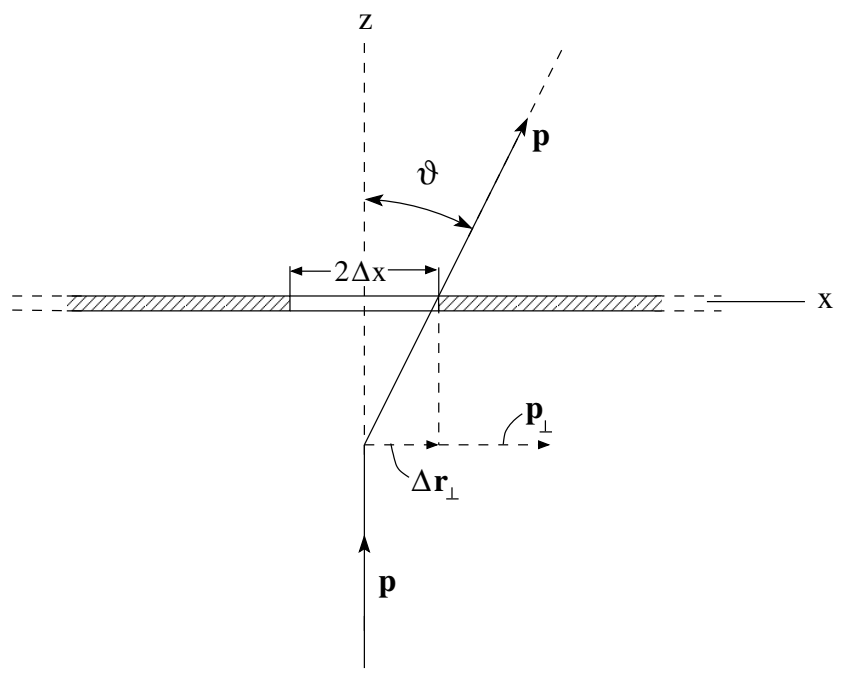

Fig. 1 Scattering at a slit.

\section{Some consequences of vacuum fluctuations}

The objective of this section is to give a brief synopsis of how the idea of a temporary violation of classical energy conservation, which $\Delta E \Delta t \approx \hbar / 2$ refers to, can be turned into a description of the non-relativistic motion of quantum mechanical particles or their stationary state behavior.

If a free particle with a definite momentum $\mathbf{p}$ traverses the vacuum its energy $\mathbf{p}^{2} / 2 m_{0}$ undergoes temporary changes $\Delta E=\left(2 \mathbf{p} \cdot \Delta \mathbf{p}+\Delta \mathbf{p}^{2}\right) / 2 m_{0}$ that cause changes of its momentum in all directions. Those with components perpendicular to $\mathbf{p}$ lead to shifts $\Delta \mathbf{r}_{\perp}$ sideways to its previous trajectory. After $\Delta t$ seconds the original momentum (and energy) is restored so that one can regard the whole process as a reversible scattering, which, however, leads to a shift $\Delta \mathbf{r}_{\perp}$. Since the subsequent shifts can have both signs, only their square remains non-zero on averaging over sufficiently many reversible scattering processes of this kind. This average can be obtained by again invoking $\Delta E \Delta t \approx \hbar / 2$ and setting $\Delta E=\Delta \mathbf{p}_{\perp}^{2} / 2 m_{0}=$ $\frac{1}{2} m_{0}\left(\Delta \mathbf{v}_{\perp}\right)^{2}$ which yields $\hbar \Delta t / 2 \approx \frac{1}{2} m_{0}\left(\Delta \mathbf{v}_{\perp} \Delta t\right)^{2}$.

We observe that $\Delta \mathbf{v}_{\perp} \Delta t=\Delta \mathbf{r}_{\perp}$, and hence

$$
\Delta \mathbf{r}_{\perp}^{2} \approx \frac{\hbar}{m_{0}} \Delta t .
$$

To keep the notation simple we have omitted overlines here to characterize the quantities as averages.

We may now define a cone which is rotational symmetric around the particle's unperturbed (classical) trajectory and has a cross section of $\sqrt{\frac{4 \hbar \Delta t}{m_{0}}}$ in diameter at the classical position of the particle. It comprises essentially all sections of the perturbed trajectory and its cross section widens in time as the particle moves further. If the particle is very fast and heavy and if its trajectory as a free particle is confined to a certain section, the diameter of this cone remains small everywhere and can well prove undetectable in practice. The images of $\alpha$-particle trajectories in a cloud chamber are well known examples of this situation. In the case of a macroscopical particle, a steel ball, for example, which drops in a vacuum chamber, it seems to be clear that there will be no aberration from its classical trajectory. However, on a microscopic scale the trajectory of its center of gravity is irregular as well, but the diameter of the cone remains tiny everywhere because $m_{0}$ is large.

We now consider a particle that is on its way through the middle of a slit of width $2 \Delta x$ cut into some planar material of "infinitesimally small" thickness. This situation is illustrated in Fig. 1. Due to a vacuum fluctuation the particle may receive an extra momentum $\Delta \mathbf{p}_{\perp}$ across its original momentum before it reaches the slit. This momentum must not be redelivered to the vacuum at the end of the scattering process because 
the vacuum can instead transfer a momentum $-\Delta \mathbf{p}_{\perp}$ to the wall material of the slit next to the particle. The momentum of the particle/wall system perpendicular to the particle's original motion is restored by this. However, to restore the original energy as well, the particle must in addition transfer the momentum

$$
\Delta \mathbf{p}_{\|}=\mathbf{p}\left(1-\sqrt{1-\left(\Delta p_{\perp} / p\right)^{2}}\right)
$$

to the wall where $\Delta \mathbf{p}_{\|}$is in the direction of the original momentum $\mathbf{p}$. Thereby the total momentum of the particle/wall system is reestablished. After leaving the slit the particle now moves on a deflected trajectory. As it was originally moving on a trajectory through the middle of the slit and, after having received an extra momentum $\Delta \mathbf{p}_{\perp}$, moved sideways by $\Delta r_{\perp}=\Delta x$ toward the edge of the slit, the duration $\Delta t$ of the energy fluctuation is given by

$$
\frac{\Delta \mathbf{p}_{\perp}^{2}}{2 m_{0}} \Delta t \approx \frac{1}{2} \hbar .
$$

Because of $\Delta \mathbf{v}_{\perp}=\Delta \mathbf{p}_{\perp} / m_{0}$ and $\Delta \mathbf{r}_{\perp}=\Delta \mathbf{v}_{\perp} \Delta t$ we thus have

$$
\Delta p_{\perp} \Delta x \approx \hbar \quad \text { and } \quad \sin \vartheta=\frac{\Delta p_{\perp}}{p}=\frac{\hbar}{p \Delta x}
$$

where $\vartheta$ is the deflection angle. That means, the deflection angle increases as the slit narrows, but it decreases if the particle becomes faster. This is in agreement with the observations. It is also obvious from the above argument that the particle in question must not be required to be a point mass. Beams of large particles like fullerene molecules which have recently been studied in the context of deflection at slits (s. Zeilinger and associates [26]), behave the same way as electrons, for example.

Employing the same concept of vacuum fluctuations that temporarily change the energy of a particle, yields an immediate understanding of the stability of atoms in their ground-state. We confine ourselves here to considering only hydrogen as the simplest case of an atom. Without vacuum fluctuations effecting the motion of the electron, the latter would eventually attain a position $\approx 10^{-13} \mathrm{~cm}$ from the nucleus where its potential energy becomes comparable to the creation energy of electron-positron pairs. Because of lepton number conservation it does not merge with the proton. We denote its energy at this distance by $E(0)$, the magnitude of which, however, will be of no importance to the following considerations.

Let a vacuum fluctuation spontaneously transfer a radial momentum to the electron so that it can reach a distance $R$ from the nucleus at which it has fully used up its kinetic energy and come to a stop. After the elapse of a total time of $\Delta t$ the electron has moved to $R$ and back to its previous position, thereby restoring its original energy $E(0)$. Within this time interval the motion of the electron is governed by

$$
\frac{m_{0}}{2} \dot{r}^{2}=\frac{e^{2}}{4 \pi \varepsilon_{0}}\left(\frac{1}{r}-\frac{1}{R}\right)
$$

where $r$ is the remaining distance from the nucleus which in the present situation is assumed to be a point charge. The quantity $\varepsilon_{0}$ denotes the capacity of the vacuum.

We rewrite the above differential equation in the form

$$
\dot{r}=\frac{1}{\beta} \sqrt{\frac{1}{r}-\frac{1}{R}}
$$

where

$$
\beta=\frac{1}{e} \sqrt{2 \pi m_{0} \varepsilon_{0}}
$$

or alternatively

$$
\frac{d t}{d r}=\beta R^{1 / 2} \frac{r^{1 / 2}}{\sqrt{R-r}} .
$$


The average kinetic energy is given by

$$
\bar{E}_{\text {kin. }}=\frac{2}{\Delta t} \int_{0}^{\Delta t / 2} \frac{m_{0}}{2} \dot{r}^{2}\left(t^{\prime}\right) d t^{\prime}=\frac{e^{2}}{4 \pi \varepsilon_{0}} \frac{2}{\Delta t} \int_{0}^{R}\left(\frac{1}{r}-\frac{1}{R}\right) \frac{d t}{d r} d r .
$$

In the second integral we have replaced the lower limit of $\approx 10^{-13} \mathrm{~cm}$ by zero. If one inserts here $\frac{d t}{d r}$ from above, the evaluation of that integral yields

$$
\bar{E}_{\mathrm{kin} .}=\frac{1}{\Delta t} \frac{e^{2}}{4 \pi \varepsilon_{0}} \pi \beta \sqrt{R} .
$$

The result may be rewritten

$$
\bar{E}_{\text {kin. }} \Delta t=\frac{e^{2}}{4 \pi \varepsilon_{0}} \pi \beta \sqrt{R} .
$$

As $\bar{E}_{\text {kin. }}$ stands for the average energy fluctuation $\Delta E$, we have

$$
\Delta E \Delta t=f \hbar
$$

where $f$ is a dimensionless factor whose lower limit is $1 / 2$, but there is otherwise a certain latitude in its value. The latter equation together with the preceding one yields

$$
R=\frac{2 f^{2}}{\pi^{2}} \frac{\hbar^{2}}{m_{0} e^{2}} 4 \pi \varepsilon_{0}=\frac{2 f^{2}}{\pi^{2}} r_{\mathrm{B}}
$$

where

$$
r_{\mathrm{B}}=\frac{\hbar^{2}}{m_{0} e^{2}} 4 \pi \varepsilon_{0}
$$

is the Bohr radius. If one chooses

$$
f=2.2
$$

$R$ becomes identical with the Bohr radius.

It is hence obvious, that the size of the hydrogen atom in its ground-state can well be explained by vacuum fluctuations which scatter the electron in an irregular fashion from its position near the nucleus out to distances of approximately $r_{\mathrm{B}}$.

As already stated earlier, the occurrence of zero-point motion with harmonic oscillators is another example of the presence of vacuum fluctuations. If a particle moves along the x-axis in a potential

$$
U(x)=\frac{m_{0}}{2} \omega_{0}^{2} x^{2}
$$

where it could classically oscillate with the frequency $\omega_{0}$, the particular situation where it is classically at rest at $x=0$ cannot persist under vacuum fluctuations. The latter impart irregularly spontaneous momenta $\mathbf{p}$ on the particle which enable it to move up to a turning point $x_{t} \neq 0$ so that

$$
U\left(x_{t}\right)=\Delta E \approx \frac{\hbar}{\Delta t} .
$$

Here $\Delta t$ denotes the time needed by the particle to reach the point $x_{t}$ and move back to its rest position which amounts to half of the oscillation period. Hence we have $\Delta t=\frac{\pi}{\omega_{0}}$ which on insertion into the above equation yields

$$
\Delta E=\frac{\hbar \omega_{0}}{\pi}
$$




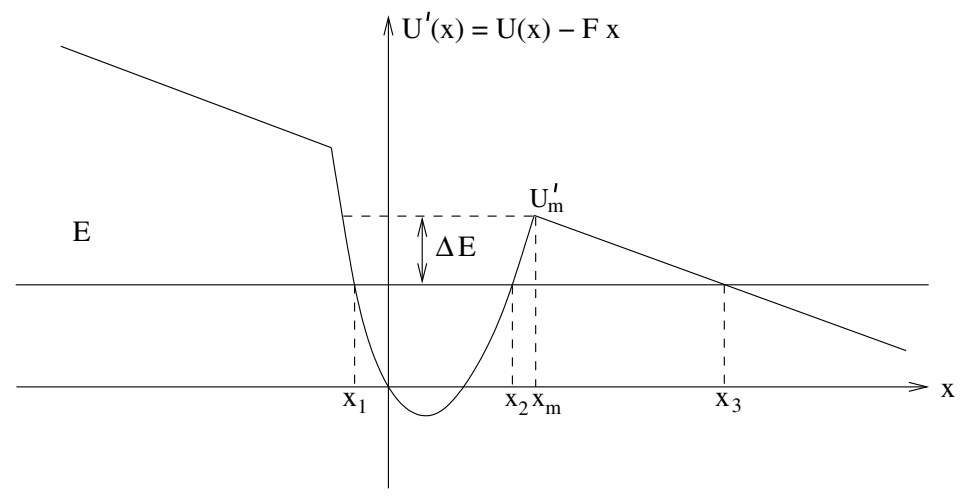

Fig. 2 Harmonic oscillator in a uniform electric field.

The exact result derived from the Schrödinger equation yields $\frac{\hbar \omega_{0}}{2}$. This energy is equal to twice the average potential energy, that is

$$
\bar{U}=\frac{m_{0}}{2} \omega_{0}^{2} \overline{x^{2}}=\frac{1}{2} \frac{\hbar \omega_{0}}{2}
$$

where $\overline{x^{2}}$ is the mean square distance of the atom from its equilibrium point. The square root of $\overline{x^{2}}$ defines the diameter $d_{0}$ of the space absorbed by the oscillating atom in its state of lowest energy. It follows then from the above equation that

$$
d_{0}=\sqrt{\frac{\hbar}{2 m_{0} \omega_{0}}} .
$$

If one considers solid $\mathrm{He}^{4}$ under pressure as a regular array of harmonic oscillators, each oscillator referring to an individual atom moving around a classical equilibrium position, this array can only be stable as long as

$$
d_{0} \ll d
$$

where $d$ is the diameter of a $\mathrm{He}^{4}$ atom. As one lowers the pressure, $\omega_{0}$ decreases and $d_{0}$ will eventually no longer fulfill the above inequality. As a conseqence, the lattice melts. As $\mathrm{He}^{4}$ is experimentally known to be a liquid at normal pressure, the occurrence of solidification can hence be understood by reversing the above argument.

The so-called quantum mechanical tunneling effect can be explained much along the lines of the preceding examples: we consider a charged particle in a potential as depicted in Fig. 2. Obviously, the potential consists of a parabolic trough onto which a linear potential has been superimposed that describes an external uniform field $\widehat{\mathbf{E}}=\mathbf{F} / e$ where $\mathrm{e}$ is the charge of the particle moving in that potential and $\mathbf{F}$ is the force exerted on the particle by the external electric field. The constant energy E of the particle is characterized by a straight horizontal line. Without the external field there would be intersections of the oscillator potential and this line at $x_{1}, x_{2}$, but - different from the situation shown in the Figure - they would be shifted to the left as the minimum of the parabola which would lie then at $x=0$ and move up to the origin. These intersections mark turning points of the classical motion of the particle. The occurrence of a third point of intersection, $x_{3}$, that appears in the presence of the external field, does not have any effect on the particle as long as it moves classically. However, under the influence of vacuum fluctuations the particle can reach this point if its temporary energy enhancement $\Delta E$ is such that

$$
\Delta E=U_{\mathrm{m}}-E \approx \frac{\hbar}{\Delta t},
$$


that is

$$
\Delta t=\frac{\hbar}{U_{\mathrm{m}}-E}
$$

where $\Delta t$ is the time interval within which the particle has to return to its "points of energy conservation" $x_{2}$ or $x_{3}$. The maximum of the potential has been denoted by $U_{\mathrm{m}}$, its coordinate by $x_{\mathrm{m}}$. To reach the point $x_{3}$, its classical motion from $x_{\mathrm{m}}$ to $x_{3}$ must be performed within $\Delta t$ seconds which means

$$
x_{3}-x_{\mathrm{m}}=\frac{F}{2 m_{0}}(\Delta t)^{2} .
$$

Inserting $\Delta t$ from the previous equation one obtains

$$
x_{3}-x_{\mathrm{m}} \leq \frac{F}{2 m_{0}}\left(\frac{\hbar}{\Delta E}\right)^{2} .
$$

The " $\leq$ "-sign refers to a modification of our assumption concerning $\Delta t$ : the latter should be the time a particle needs at most to travers the distance from $x_{\mathrm{m}}$ to $x_{3}$.

We now exploit the fact that $\Delta E /\left(x_{3}-x_{\mathrm{m}}\right)$ represents - except for the sign - just the gradient of the linear portion of the potential, which means that it is identical with $-F$. Hence

$$
x_{3}-x_{\mathrm{m}}=\frac{\Delta E}{F} .
$$

This combined with the foregoing equation yields

$$
\frac{\sqrt{2 m_{0}}}{\hbar F} \phi^{\frac{3}{2}} \leq 1 \quad \text { where } \quad \phi=U_{\mathrm{m}}-E .
$$

The latter inequality represents a condition for the magnitude of the electric field $\widehat{\mathbf{E}}=\mathbf{F} / e$ to allow the particle to escape from the trough. We have replaced $U_{\mathrm{m}}-E$ by $\phi$ which represents the "work function" of the system, i. e. the minimum energy needed to extract an electron from a metal, for example.

The above result contains the essential interconnection of quantities governing the emission of particles from a metal tip. It is this combination of quantities which appears in the Fowler-Nordheim formula describing the emission of particles from a metal tip under the influence of an external electric field.

But the above simple derivation yields an additional interesting information which is otherwise not easily accessible. The quantity $\Delta t$ given by $\Delta t=\hbar /\left(U_{\mathrm{m}}-E\right)=\hbar / \phi$ describes the time needed by the particle to leave the trough. Because of

$$
\hbar=6.6 \cdot 10^{-16} \mathrm{eVs} \text { and } \phi \approx 5 \mathrm{eV}
$$

the passage times are of the order of $10^{-16} \mathrm{~s}$.

One can easily verify that $\sqrt{2 m_{0}} \phi^{3 / 2} / \hbar F$ equals unity at an electric field strength of $\widehat{E} \approx 3 \cdot 10^{8} \mathrm{~V} \mathrm{~cm}^{-1}$ if $\phi \approx 4.6 \mathrm{eV}$ which is the work function of tungsten metal and also the approximate magnitude of the work function of many other transition metals. If one takes the tungsten tip of a typical field electron microscope as an example where the emitting tip is essentially a half-sphere of radius $R \approx 5 \cdot 10^{-6} \mathrm{~cm}$ field emission occurs at a voltage of $V \approx 1.5 \mathrm{kV}$ since $\widehat{E}=V / R$. This is exactly what one observes.

It is obvious from the above considerations that the term "tunneling effect" is actually misleading if not downright wrong, because in actual fact the particle has to overcome the potential maximum at $x_{\mathrm{m}}$ if it succeeds in escaping the trough. Only from a classical point of view the emission of the particle seems to have been accomplished by "drilling a tunnel" through the potential barrier at a distance of $U_{\mathrm{m}}-E$ from the top. 
We now turn to the problem of how to describe the motion of a particle whose energy fluctuates irregularly around its constant average according to $\Delta E \Delta t \approx \hbar / 2$ and gradually drifts away from its classical trajectory. Actually, this cannot be a meaningful objective because it implies the possibility that the point at which the trajectory starts can physically be well defined. At first sight it seems that this can easily be realized: one would only need a source, for example, an atomic size tip of a tunneling microscope which ejects electrons of a certain absolute value of momentum, and a pinhole in front of the source so that only one trajectory can pass through that hole. However, as explained above, the particle would strongly be deflected by the wall material of the pinhole and with overwhelming probability not continue its motion in the original direction. Consequently, to avoid any non-classical perturbation of its trajectory, the hole must be very large in diameter, and the trajectories of the particles that are radially ejected from the tip within a solid angle of $\Omega \leq 2 \pi$ must be bundled by an electron lense to form a beam of macroscopic cross section such that the momentum $\mathbf{p}$ of each particle, averaged over many reversible scatterings, is the same everywhere in the beam, irrespective of the particle one chooses. Hence, a consistent description of the motion of a particle must comprise the entire space that is accessible due to vacuum fluctuations and because the initial lateral position of the particle (in the present example: the starting point at the surface of the atom at the tip) is only probabilistically determined when the particle starts moving along its irregular trajactory. The same applies to a particle that is bound in an attractive potential where it can be scattered to any position away from its classical orbit.

With this view in mind we subdivide the accessible space of volume $V$ into $N_{\mathrm{c}}$ sufficiently small cubes $\Delta^{3} r_{\nu}$ numbered by an index $\nu$ and focus on one of the cubes labeled by $\nu_{0}$. We imagine that we were able to mimic the irregular trajectory of a particle, that undergoes reversible scatterings, in a computer simulation and perform this simulation in parallel for a very large number of $N$ equivalent systems which only differ in the starting points of their trajectories and in the time at which they start their motion. We then draw all trajectories together in a three-dimensional visualization which yields a family of very densely packed trajectories all of which represent equivalent particle histories. It is this ensemble which we shall henceforth refer to. In the following it will be convenient to introduce a function for the $j$-th particle

$$
\varepsilon_{j}\left(\overline{\mathbf{r}}_{\nu}, t\right)=\int_{\Delta^{3} r_{\nu}} \delta\left(\mathbf{r}_{j}(t)-\mathbf{r}\right) d^{3} r= \begin{cases}1 & \text { if } \mathbf{r}_{j}(t) \text { is contained in } \Delta^{3} r_{\nu} \\ 0 & \text { otherwise }\end{cases}
$$

where $\delta\left(\mathbf{r}_{j}(t)-\mathbf{r}\right)$ stands for the Dirac function and $\overline{\mathbf{r}}_{\nu}$ denotes some point in $\Delta^{3} r_{\nu}$. As we are considering only cubes of very small size, we may justifiably replace $\overline{\mathbf{r}}_{\nu}$ by $\mathbf{r}$ from now on where $\mathbf{r}$ is always meant to lie within the cube $\Delta^{3} r_{\nu_{0}}$ under consideration. We may now define an ensemble average of the particle velocity by

$$
\mathbf{v}(\mathbf{r}, t)=\frac{\sum_{j=1}^{N} \varepsilon_{j}(\mathbf{r}, t) \mathbf{v}_{j}(t)}{\sum_{j=1}^{N} \varepsilon_{j}(\mathbf{r}, t)}
$$

and, likewise, a probability density of finding a particle in $\Delta^{3} r_{\nu_{0}}$

$$
\rho(\mathbf{r}, t)=\frac{\Delta N(\mathbf{r}, t)}{\Delta^{3} r_{\nu_{0}}}
$$

where

$$
\Delta N(\mathbf{r}, t)=\frac{1}{N} \sum_{j=1}^{N} \varepsilon_{j}(\mathbf{r}, t) \quad \text { and } \quad \sum_{\nu=1}^{N_{\mathrm{c}}} \Delta N\left(\mathbf{r}_{\nu}, t\right)=1 .
$$

Obviously, $\rho(\mathbf{r}, t)$ has the property

$$
\int_{V} \rho(\mathbf{r}, t) d^{3} r=1
$$


In a stationary state situation the functions $\rho(\mathbf{r}, t)$ and $\mathbf{v}(\mathbf{r}, t)$ are actually time-independent. However, if the initial conditions or the potentials are time-dependent, the probability density and the average velocity are time-dependent as well. As in the case of a hydrodynamical gaseous system that consists of atoms or atomic composites, the trajectory of an individual particle is definitely very irregular but still differentiable, otherwise the stochastic forces, which are responsible for the irregularity, would not be defined everywhere. But the ensemble average $\mathbf{v}(\mathbf{r}, t)$ will be a smoothly varying function again. With these definitions at hand, we are now in the position to elucidate Nelson's idea of incorporating vacuum fluctuations into a Newtonian theory of particle motion. His hypothesis is as follows: the vacuum induced energy changes $\Delta E$ of a particle, which last for an average time interval $\Delta t$ so that $\Delta E \Delta t \approx \hbar / 2$, can be simulated by assuming that the particle performs a modified Brownian motion driven by fluctuating vacuum forces. That means more specifically: one subdivides the system of $N$ independent particles, defining the fundamental ensemble, into two subsets B (for "Brownian") and A (for "Anti-Brownian"). These subsystems are subject to stochastic forces $\mathbf{F}_{s}^{\mathrm{A} / \mathrm{B}}(t)$ which can generally be decomposed into a random force $\mathbf{F}_{R}(t)$ with a Gaussian probability distribution around zero acting equally in both subsytems (i.e. $\mathbf{F}_{R}^{\mathrm{A}}(t)=\mathbf{F}_{R}^{\mathrm{B}}(t)$ ) and into a mean force proportional to the particle velocity $\mathbf{v}_{j}(t)$. That mean force is $-\frac{m_{0} \mathbf{v}_{j}(t)}{\tau}$ for the B-system, and hence slows down the motion of the particle within $\tau$ seconds if it were free, and it is $+\frac{m_{0} \mathbf{v}_{j}(t)}{\tau}$ for the A-system implying an enhancement of the A-particle motion. The two subsystems are characterized by pairs of functions $\left\{\rho_{\mathrm{B}}(\mathbf{r}, t), \mathbf{v}_{\mathrm{B}}(\mathbf{r}, t)\right\}$ and $\left.\left\{\rho_{\mathrm{A}}(\mathbf{r}, t)\right\}, \mathbf{v}_{\mathrm{A}}(\mathbf{r}, t)\right\}$, and the subdivision is performed such that

$$
\rho_{\mathrm{A}}(\mathbf{r}, t)=\rho_{\mathrm{B}}(\mathbf{r}, t) \quad \text { and } \quad \mathbf{v}_{\mathrm{A}}(\mathbf{r}, t)=\mathbf{v}_{\mathrm{B}}(\mathbf{r}, t) .
$$

Note that is always possible to ensure $\mathbf{v}_{\mathrm{B}}(\mathbf{r}, t)=\mathbf{v}_{\mathrm{A}}(\mathbf{r}, t)$. If a tentative subdivision does not comply with that requirement and one would have $\mathbf{v}_{\mathrm{B}}(\mathbf{r}, t)<\mathbf{v}_{\mathrm{A}}(\mathbf{r}, t)$, for example, one can interchange particles from $\mathrm{B}$ and $\mathrm{A}$ (slow ones from $\mathrm{B}$ for as many, but faster ones from $\mathrm{A}$ ) so that one finally obtains equality of the velocities without having changed the number of particles in A and B. This possibility of interchanging particles $\mathrm{A}$ and $\mathrm{B}$ to equalize the velocities $\mathbf{v}_{\mathrm{A}}$ and $\mathbf{v}_{\mathrm{B}}$ will be of great importance to the following.

We consider the temporal changes within a time interval $\Delta t \geq \tau$ that occur within $\Delta^{3} r_{\nu_{0}}$ due to particle transitions from or to the cubes outside. The B-system will lose energy as opposed to the A-system whose energy increases. Hence, if one averages over the two rates of changes one obtains a differential equation that describes a system without energy dissipation. Since the time-evolution of the two subsystems diverges, one has to redivide the whole system of $N$ particles into subsystems B and A for the subsequent time step $\Delta t$ by newly decomposing the set of $n_{\nu}$ particles in $\Delta^{3} r_{\nu_{0}}$ into $n_{\nu} / 2$ particles for $\mathrm{B}$ and $n_{\nu} / 2$ particles for A such that $\mathbf{v}_{\mathrm{A}}(\mathbf{r}, t)=\mathbf{v}_{\mathrm{B}}(\mathbf{r}, t)$ with $t$ now referring to the new time interval. Hence, if one follows a specific particle along its irregular trajectory it will as often belong to the B-system as to the A-system on the average. As this change from B- to A-character of a particle is not described by the transition probabilities governing the time evolution of, respectively, the A- and the independent B-system within a certain time step $\Delta t \geq \tau$, the overall process described by the above procedure is non-Markovian. This has clearly been pointed out also by M. Requardt [27].

It is, furthermore, important to note that temporal changes of such stochastic systems, which are formally described by differential equations, are actually taking place on a coarse grained time scale which means that the time $t$ is defined only within a certain margin $\tau$.

The artificial subdivision of the original ensemble, comprising $N$ particles, into A- and B-systems, where members are interchanged to reestablish equality of the velocities $\mathbf{v}_{\mathrm{A}}$ and $\mathbf{v}_{\mathrm{B}}$, is only an auxiliary trick to mimic what happens naturally to a single particle under the influence of vacuum fluctuations. But this particular picture provides a simple rationale for what causes the outcome of the two-slit experiment: as a consequence of interchanging B- and A-particles after each time step the trajectories of the particles become intertwined. That means: a particle that has moved through the first slit and reached the cube $\Delta^{3} r_{\nu_{0}}$ at some distance from the slit as a "B-particle", may be interchanged with a particle that moved through the second slit if the trajectory of the latter happens to enter the same cube as an "A-particle". If the second slit is closed, the B-particle's further trajectory will be different because certain choices of interchange do not 
exist any more. It is evident from this example that the process of interchanging particles which ensures the absence of dissipation, leads automatically to a non-locality in the description of the particle motion. However, one should always keep in mind that one is actually dealing with a single-particle system whose motion is controlled by certain scattering probabilities. The latter have to ensure reversibility, which means: if the particle has changed its momentum at some point in real space, the vacuum has to remember at a different "point of restauration" what exactly the previous values of energy and momentum had been. Only to mimic these particular scattering probabilities one resorts to the behavior of our artificial ensemble, that is, to the behavior of two subsets of independent particles which represent different histories under equivalent classical conditions. This will become apparent in the discussion of the Smoluchowski equation in Sect. 4.

It is clear that a particle whose probability of being at various positions in space is governed by the Schrödinger equation, which we shall derive, is under no circumstances a wave. This is in analogy to a Brownian particle whose probability in space is governed by the equation of diffusion, but is never a continuous medium. The "wave/particle duality"-parlance is unacceptable in a stochastic quantum theory of particle motion because this parlance pretends to describe something which actually does not exist.

In concluding this Section we want to comment on a recent article by Hall and Reginatto [28] which also presents a derivation of the one-particle Schrödinger equation. This paper is based on the concept of random momentum fluctuations of the particle which the authors require to inversely correlate with the position fluctuations such that $\Delta p_{i} \Delta x_{i}(i=1,2,3)$ denoting the coordinate axes) is preserved under a scaling transformation $x_{i} \rightarrow \alpha x_{i} ; p_{i} \rightarrow \alpha p_{i}$ where $\alpha$ is real-valued und dimensionless and $\Delta p_{i}, \Delta x_{i}$ denote rms-values of the respective fluctuations.

The authors fail to associate these momentum fluctuations with energy fluctuations which we consider to be of vital importance to an immediate understanding of the stability of atoms and the tunneling effect. As the authors, unfortunately, deem it a particular virtue of their approach that it effectively eliminates the notion of particle trajectories, they discard the helpful concept of following a particle along its path of reversible scatterings. But only this concept reveals the memory effect of the vacuum which transforms into the interference properties of the associated wavefunction at a later stage of our derivation. Furthermore, our qualitative explanation of the two-slit experiment was intimately tied to the existence of a particle trajectory and to its probability of continuing in a certain direction at a certain point. This probability depends on whether or not the second slit is open, even when it has not passed through that slit.

We advance the opinion that our adaptation of Nelson's approach has definitely more to offer in terms of physical insight.

\section{Brownian motion}

We start with first considering the Brownian subsystem. As pointed out in the foregoing Section, we describe the effect of the vacuum on the $j$-th particle by a stochastic force

$$
\mathbf{F}_{s j}^{\mathrm{B}}(t)=\mathbf{F}_{r j}^{\mathrm{B}}(t)-\frac{m_{0}}{\tau} \mathbf{v}_{j}(t)
$$

where $\mathbf{v}_{j}$ is the particle velocity and $\mathbf{F}_{r}^{\mathrm{B}}=\left(F_{r 1}, F_{r 2}, F_{r 3}\right)$ denotes a random force with a Gaussian distribution

$$
P\left(F_{r k}^{\mathrm{B}}\right)=\frac{1}{\sqrt{\pi} F_{r 0}} e^{-\left(F_{r k}^{\mathrm{B}} / F_{r 0}\right)^{2}} \quad(k=1,2,3) .
$$

The quantity $F_{r 0}$ is defined

$$
F_{r 0}=\frac{1}{\tau_{\text {coll. }}} \sqrt{m_{0} k_{\mathrm{B}} T}
$$

where it has been assumed that the "vacuum" can be assigned an effective temperature $T$. The quantity $k_{\mathrm{B}}$ denotes the Boltzmann constant, and $\tau_{\text {coll. }}$ describes a mean time of momentum transfer. Hence eq. (4) can 
alternatively be cast as

$$
\frac{\overline{\Delta p_{k}^{2}}}{2 m_{0}}=\frac{1}{2} k_{\mathrm{B}} T \longrightarrow \frac{\overline{\Delta \mathbf{p}^{2}}}{2 m_{0}}=\frac{3}{2} k_{\mathrm{B}} T
$$

where $\overline{\Delta p_{k}^{2}}$ is the mean square of the momentum transfer.

To avoid gaps in the detailed understanding of our considerations we restate some essential steps in the derivation of Einstein's law [29] on the mean square displacement of the particle.

The equation of motion for the $j$-th particle takes the form of Langevin's equation [30]

$$
m_{0} \ddot{\sigma}_{j k}+\frac{m_{0}}{\tau} \dot{\sigma}_{j k}=F_{j k}+F_{r j k}^{\mathrm{B}}(t)
$$

where $\underline{\sigma}_{j}=\left(\sigma_{1}, \sigma_{2}, \sigma_{3}\right)$ describes the shift of the particle's position and

$$
\mathbf{F}_{j}=-\nabla V\left(\mathbf{r}_{j}\right)
$$

is the external classical force acting on the particle, $V(\mathbf{r})$ the associated potential. The shift $\underline{\sigma}_{j}$ may be subdivided into a portion $\underline{\sigma}_{r j}$ that is caused by the random force, and a portion $\underline{\sigma}_{c}$ that occurs when the random force is absent. The subscript "c" stands for "convection". Hence, these shifts obey equations

$$
\ddot{\sigma}_{r j k}+\frac{1}{\tau} \dot{\sigma}_{r j k}=f_{r j k}^{\mathrm{B}}
$$

and

$$
\ddot{\sigma}_{c j k}+\frac{1}{\tau} \dot{\sigma}_{c j k}=f_{j k}
$$

where

$$
f_{r j k}^{\mathrm{B}}=\frac{1}{m_{0}} F_{r j k}^{\mathrm{B}} ; \quad f_{j k}=\frac{1}{m_{0}} F_{j k} .
$$

Obviously, the sum of eqs. (8) and (9) yields eq. (6).

Muliplication of eq. (8) by $\sigma_{r j l}$ gives

$$
\frac{d}{d t}\left(\sigma_{r j l} \dot{\sigma}_{r j k}\right)-\dot{\sigma}_{r j l} \dot{\sigma}_{r j k}=-\frac{1}{\tau} \sigma_{r j l} \dot{\sigma}_{r j k}+\sigma_{r j l} f_{r j k}^{\mathrm{B}}
$$

where we have observed that

$$
\frac{d}{d t}\left(\sigma_{r j l} \dot{\sigma}_{r j k}\right)=\sigma_{r j l} \ddot{\sigma}_{r j k}+\dot{\sigma}_{r j l} \dot{\sigma}_{r j k} .
$$

We now form the ensemble average of eq. (10) in analogy to eq. (1) in Sect. 2. As there is no correlation between $\sigma_{r j l}$ and $f_{r j k}^{\mathrm{B}}$ including $l=k$, we have

$$
\frac{1}{\sum_{j=1}^{N} \varepsilon_{j}(\mathbf{r}, t)} \sum_{j=1}^{N} \varepsilon_{j}(\mathbf{r}, t) \sigma_{r j l} f_{r j k}^{\mathrm{B}}=0 .
$$

There is also no correlation between $\dot{\sigma}_{r j l}$ and $\dot{\sigma}_{r j k}$ for $l \neq k$, and hence

$$
\frac{1}{\sum_{j=1}^{N} \varepsilon_{j}(\mathbf{r}, t)} \sum_{j=1}^{N} \varepsilon_{j}(\mathbf{r}, t) \dot{\sigma}_{r j l} \dot{\sigma}_{r j k}=\delta_{l k}\left[\dot{\sigma}_{r k}(\mathbf{r}, t)\right]^{2} .
$$


Because of eq. (5) we thus have

$$
\left[\dot{\sigma}_{r k}(\mathbf{r}, t)\right]^{2}=\frac{k_{\mathrm{B}} T}{m_{0}} .
$$

As a result, the ensemble average of eq. (10) takes the form

$$
\frac{\partial}{\partial t}\left(\sigma_{r l} \dot{\sigma}_{r k}\right)+\frac{1}{\tau}\left(\sigma_{r l} \dot{\sigma}_{r k}\right)=\delta_{l k} \frac{k_{\mathrm{B}} T}{m_{0}} .
$$

The solution of this equation can be cast as

$$
\sigma_{r l} \dot{\sigma}_{r k}=\delta_{l k} \frac{k_{\mathrm{B}} T \tau}{m_{0}}\left[1-C e^{-\frac{\left(t-t_{0}\right)}{\tau}}\right]
$$

where $C$ is some constant. Without loss of generality $\underline{\sigma}_{r}$ may be defined such that $\underline{\sigma}_{r}\left(\mathbf{r}, t_{0}\right)=0$. In that case $\mathrm{C}$ becomes equal to one.

For a time interval

$$
\Delta t=t-t_{0} \gg \tau
$$

the second term in the foregoing equation may be neglected, and we arrive at

$$
\sigma_{r l} \dot{\sigma}_{r k}=\delta_{l k} \nu
$$

where

$$
\nu=\frac{k_{\mathrm{B}} T \tau}{m_{0}}
$$

denotes the kinematic viscosity that the particle experiences in the embedding medium.

We observe that

$$
\sigma_{r k} \dot{\sigma}_{r k}=\frac{1}{2} \frac{\partial}{\partial t} \sigma_{r k}^{2}
$$

and integrate eq. (14) within the time interval $\left[t_{0}, t_{0}+\Delta t\right]$. The result may be written

$$
\sigma_{r l} \sigma_{r k}=\delta_{l k} 2 \nu \Delta t
$$

where we have set $\sigma_{r k}^{2}\left(\mathbf{r}, t_{0}\right)=0$ in agreement with our assumption on $\underline{\sigma}_{r}\left(\mathbf{r}, t_{0}\right)$ above.

Eq. (16) represents Einstein's famous law on the time dependence of the mean square diplacement of a particle driven by a random force with a Gaussian distribution in a viscous medium.

The total displacement of the particle, $\underline{\sigma}_{j}$, is given by $\underline{\sigma}_{r j}+\underline{\sigma}_{c j}$. When we form the ensemble average of $\sigma_{j l} \sigma_{j k}$ and exploit the fact that $\sigma_{c j l}$ and $\sigma_{r j k}$ are definitely uncorrelated, we may write the result

$$
\begin{aligned}
\sigma_{l} \sigma_{k} & =\frac{1}{\sum_{j=1}^{N} \varepsilon_{j}(\mathbf{r}, t)} \sum_{j=1}^{N} \varepsilon_{j}(\mathbf{r}, t)\left(\sigma_{c j l}+\sigma_{r j l}\right)\left(\sigma_{c j k}+\sigma_{r j k}\right) \\
& =\sigma_{c l} \sigma_{c k}+\sigma_{r l} \sigma_{r k} .
\end{aligned}
$$

Below we shall be concerned with time-dependencies within intervals $\Delta t$ that are small but still large enough to comply with the requirement (13). Without loss of generality we may also set $\sigma_{c k}\left(\mathbf{r}, t_{0}\right)=0$ and then expand $\sigma_{c k}\left(\mathbf{r}, t_{0}+\Delta t\right)$ only to linear order in $\Delta t$ :

$$
\sigma_{c k}\left(\mathbf{r}, t_{0}\right)=\dot{\sigma}_{c k}\left(\mathbf{r}, t_{0}\right) \Delta t .
$$


As $\sigma_{c l}$ and $\sigma_{c k}$ are uncorrelated for $l \neq k$, eq. (17) may be given the form

$$
\sigma_{l} \sigma_{k}=\delta_{l k}\left(2 \nu \Delta t+\dot{\sigma}_{c k}^{2} \Delta t^{2}\right)
$$

where eqs. (16) and (18) have been used. This result may be cast in a form that will prove particularly suited for the ensuing considerations.

Let $P^{\mathrm{B}}(\mathbf{r}, \underline{\sigma}, t, \Delta t) d^{3} \sigma$ be the probability of finding a particle after the elapse of time $\Delta t$ in $d^{3} \sigma$ around a position $\mathbf{r}+\underline{\sigma}$ if it was definitely at $\mathbf{r}$ at time $t$. This probability is normalized

$$
\int P^{\mathrm{B}}(\mathbf{r}, \underline{\sigma}, t, \Delta t) d^{3} \sigma=1 \text {. }
$$

Using these properties we obtain

$$
\begin{aligned}
\frac{1}{\sum_{j=1}^{N} \varepsilon_{j}(\mathbf{r}, t)} \sum_{j=1}^{N} \varepsilon_{j}(\mathbf{r}, t) \sigma_{j l}(t+\Delta t) \sigma_{j k}(t+\Delta t) & =\sigma_{l}(\mathbf{r}, t+\Delta t) \sigma_{k}(\mathbf{r}, t+\Delta t) \\
& =\int \sigma_{l} \sigma_{k} P^{\mathrm{B}}(\mathbf{r}, t, \Delta t) d^{3} \sigma
\end{aligned}
$$

and hence eq. (19) can be cast as

$$
\int \sigma_{l} \sigma_{k} P^{\mathrm{B}}(\mathbf{r}, \underline{\sigma}, t, \Delta t) d^{3} \sigma=\delta_{l k}\left(2 \nu \Delta t+\dot{\sigma}_{c k}^{2} \Delta t^{2}\right)
$$

\section{The Fokker-Planck equation}

As follows immediately from the definition of $P^{\mathrm{B}}(\mathbf{r}, \underline{\sigma}, t, \Delta t)$, the following equation holds

$$
\rho(\mathbf{r}, t+\Delta t)=\int \rho(\mathbf{r}-\underline{\sigma}, t) P^{\mathrm{B}}(\mathbf{r}-\underline{\sigma}, \underline{\sigma}, t, \Delta t) d^{3} \sigma
$$

which is known as the Smoluchowski equation [31]. Here $\rho(\mathbf{r}, t+\Delta t)$ denotes the probability density of finding the particle within the elementary cube around $\mathbf{r}$ at time $t$. This density is normalized to unity within the fundamental volume $V$.

We approximate the $\sigma$-dependence of the function

$$
G(\mathbf{r}-\underline{\sigma}, \underline{\sigma}, t, \Delta t)=\rho(\mathbf{r}-\underline{\sigma}, t) P^{\mathrm{B}}(\mathbf{r}-\underline{\sigma}, \underline{\sigma}, t, \Delta t)
$$

by a Taylor polynomial of second power

$$
G(\mathbf{r}-\underline{\sigma}, \underline{\sigma}, t, \Delta t)=G(\mathbf{r}, \underline{\sigma}, t, \Delta t)-\sum_{k=1}^{3} \sigma_{k} \frac{\partial}{\partial x_{k}} G(\mathbf{r}, \underline{\sigma}, t, \Delta t)+\frac{1}{2} \sum_{l, k} \sigma_{l} \sigma_{k} \frac{\partial^{2}}{\partial x_{l} \partial x_{k}} G(\mathbf{r}, \underline{\sigma}, t, \Delta t)
$$

and insert this expression under the integral in eq. (22). We thus obtain

$$
\begin{aligned}
\rho(\mathbf{r}, t+\Delta t)= & \rho(\mathbf{r}, t)-\sum_{k=1}^{3} \frac{\partial}{\partial x_{k}}\left[\rho(\mathbf{r}, t) \int \sigma_{k} P^{\mathrm{B}}(\mathbf{r}, \underline{\sigma}, t, \Delta t) d^{3} \sigma\right] \\
& +\frac{1}{2} \sum_{l, k} \frac{\partial^{2}}{\partial x_{l} \partial x_{k}}\left[\rho(\mathbf{r}, t) \int \sigma_{l} \sigma_{k} P^{\mathrm{B}}(\mathbf{r}, \underline{\sigma}, t, \Delta t) d^{3} \sigma\right]
\end{aligned}
$$


where we have used the normalization (20).

Because of

$$
\sigma_{k}=\sigma_{r k}+\sigma_{c k}
$$

and

$$
\int \sigma_{r k} P^{\mathrm{B}}(\mathbf{r}, \underline{\sigma}, t, \Delta t) d^{3} \sigma=0,
$$

the second integral on the rhs yields

$$
\int \sigma_{c k} P^{\mathrm{B}}(\mathbf{r}, \underline{\sigma}, t, \Delta t) d^{3} \sigma=v_{c k}(\mathbf{r}, \bar{t}) \Delta t
$$

where $\mathbf{v}_{c}$ is the convective velocity associated with $\underline{\sigma}_{c}$, and $\bar{t}$ is a suitably chosen time out of the interval $[t, t+\Delta t]$.

The third expression on the rhs of eq. (23) contains the integral (21). Dividing by $\Delta t$ we obtain in the limit $\Delta t \rightarrow 0$

$$
\frac{\partial \rho}{\partial t}+\operatorname{div}\left(\rho \mathbf{v}_{c}\right)-\nu \Delta \rho=0 .
$$

This represents the so-called Fokker-Planck equation [32] which is a special case of Kolmogorov's second differential equation [33] derived considerably later. For $\mathbf{v}_{c}=0$ eq. (24) reduces to the equation of diffusion

$$
\frac{\partial \rho}{\partial t}=\nu \Delta \rho \text {. }
$$

In deriving eq. (24) one should keep in mind that it is actually inadmissible to let $\Delta t$ go to zero because it is conflict with the coarse graining requirement (13). Eq. (24) and everything that follows should therefore taken with caution. It is exactly this point which will prove crucial in combining the behavior of the A-and the B-system to accomplish a modification of particle motion by reversible scattering.

Apart from eq. (24) the temporal changes of $\rho(\mathbf{r}, t)$ must also obey the continuity equation

$$
\frac{\partial \rho}{\partial t}+\operatorname{div} \mathbf{j}=0
$$

which preserves the number of particles of the ensemble.

We define a diffusive (or "osmotic") current density by

$$
\mathbf{j}_{\mathrm{d}}=\mathbf{j}-\mathbf{j}_{c}
$$

connected with an osmotic velocity $\mathbf{u}$

$$
\mathbf{j}_{\mathrm{d}}=\rho \mathbf{u}
$$

in analogy to

$$
\mathbf{j}=\rho \mathbf{v} \text {. }
$$

Eqs. (24) and (25) are simultaneously satisfied if

$$
\mathbf{j}_{\mathrm{d}}=-\nu \nabla \rho
$$

which is Fick's law.

From eqs. (24) to (29) we thus obtain

$$
\mathbf{v}=\mathbf{v}_{c}+\mathbf{u}
$$

and

$$
\mathbf{u}=-\nu \frac{1}{\rho} \nabla \rho .
$$




\section{The Navier-Stokes equation}

Similar to the temporal changes of $\rho(\mathbf{r}, t)$ one can calculate the changes of $\rho(\mathbf{r}, t) \mathbf{v}_{c}(\mathbf{r}, t)$ by again exploiting the properties of $P^{\mathrm{B}}(\mathbf{r}, \underline{\sigma}, t, \Delta t)$. In the absence of an external force field we have

$$
\rho(\mathbf{r}, t+\Delta t) v_{c k}(\mathbf{r}, t+\Delta t)=\int \rho(\mathbf{r}-\underline{\sigma}, t) v_{c k}(\mathbf{r}-\underline{\sigma}, t) P^{\mathrm{B}}(\mathbf{r}-\underline{\sigma}, \underline{\sigma}, t, \Delta t) d^{3} \sigma .
$$

Again, we expand the $\underline{\sigma}$-dependence of the integrand

$$
\widehat{G}(\mathbf{r}-\underline{\sigma}, \underline{\sigma} t, \Delta t)=\rho(\mathbf{r}-\underline{\sigma}, t) \mathbf{v}(\mathbf{r}-\underline{\sigma}, t) P^{\mathrm{B}}(\mathbf{r}-\underline{\sigma}, \underline{\sigma}, t, \Delta t)
$$

to first order in $\underline{\sigma}$ which, on insertion into eq. (32), yields

$$
\begin{aligned}
& \rho(\mathbf{r}, t+\Delta t) v_{c k}(\mathbf{r}, t+\Delta t)-\rho(\mathbf{r}, t) v_{c k}(\mathbf{r}, t) \\
& =-\Delta t \operatorname{div}\left(\rho v_{c k} \mathbf{v}_{\mathbf{c}}\right)+\Delta t \nu \Delta\left(\rho v_{c k}\right)+\frac{(\Delta t)^{2}}{2} \sum_{k} v_{c k}^{2} \frac{\partial^{2}\left(\rho v_{c k}\right)}{\partial x_{k}^{2}} .
\end{aligned}
$$

The expression on the lhs of this equation represents the change of the convective current density of the particle momentum at $\mathbf{r}$ within $\Delta t$ seconds. This change is solely caused by scatterings from outside cubes into the cube around $\mathbf{r}$ and vice versa. For this reason we label the partial derivative $\partial / \partial t$ which we obtain after division by $\Delta t$ and letting $\Delta t$ tend to zero by the subscript "scatt.". Hence we get

$$
\left.\frac{\partial \rho v_{c k}}{\partial t}\right|_{\text {scatt. }}=-\operatorname{div}\left(\rho v_{c k} \mathbf{v}_{\mathbf{c}}\right)+\nu \Delta\left(\rho v_{c k}\right)
$$

which analogous to eq. (24) where only $\rho$ has been replaced by $\rho v_{c k}$.

In the presence of an external force field $\mathbf{F}(\mathbf{r})$ there is an additional contribution to the change of the momentum current density given by

$$
\left.\frac{\partial \rho v_{c k}}{\partial t}\right|_{\text {force }}=\hat{f}_{k}(\mathbf{r})
$$

where

$$
\hat{f}_{k}(\mathbf{r})=\frac{1}{m_{0}} \rho(\mathbf{r}) F_{k}(\mathbf{r})
$$

denotes the mass-referenced force density. Summing the two contributions (34) and (35) we obtain

$$
\frac{\partial \rho v_{c k}}{\partial t}=-\operatorname{div}\left(\rho v_{c k} \mathbf{v}_{\mathbf{c}}\right)+\nu \Delta\left(\rho v_{c k}\right)+\hat{f}_{k}
$$

which can be recast as

$$
v_{c k}\left[\frac{\partial \rho}{\partial t}+\operatorname{div}\left(\rho \mathbf{v}_{\mathbf{c}}\right)-\nu \Delta \rho\right]+\rho\left[\frac{\partial v_{c k}}{\partial t}+\left(\mathbf{v}_{\mathbf{c}} \cdot \nabla\right) v_{c k}-\nu \Delta v_{c k}-2 \nu \frac{1}{\rho} \nabla \rho \cdot \nabla v_{c k}\right]=\hat{f}_{k}
$$

where we have used

$$
\Delta\left(\rho v_{c k}\right)=\rho \Delta v_{c k}+v_{c k} \Delta \rho+2 \nabla v_{c k} \cdot \nabla \rho
$$

and

$$
\frac{\partial \rho v_{c k}}{\partial t}=\rho \frac{\partial v_{c k}}{\partial t}+v_{c k} \frac{\partial \rho}{\partial t}
$$


Because of the Fokker-Planck equation (24) the first bracketed expression equals zero. If we, furthermore, use the definitions (31) and (36) of $\mathbf{u}$ and $\hat{f}_{k}$, respectively, eq. (37) may be rewritten

$$
\frac{\partial \mathbf{v}_{\mathrm{c}}}{\partial t}+\left(\mathbf{v}_{c}+2 \mathbf{u}\right) \cdot \nabla \mathbf{v}_{c}-\nu \Delta \mathbf{v}_{c}=\frac{1}{m_{0}} \mathbf{F}(\mathbf{r})
$$

It should be noticed that the above procedure amounts to replacing the transtion probability $P^{\mathrm{B}}(\mathbf{r}-$ $\underline{\sigma}, \underline{\sigma}, t, \Delta t)$ by expressions of $\mathbf{v}_{c}$ and $\mathbf{u}$ which contain equivalent information about the ensemble.

In a fluid medium with a mass density $\widehat{\rho}(\mathbf{r}, t)=m_{0} \rho(\mathbf{r}, t)$ the force acting on the elementary cube is given by

$$
\rho \mathbf{F}(\mathbf{r}, t)=-\nabla p(\mathbf{r}, t)
$$

where $p$ is the pressure. In practice, the osmotic velocity $\mathbf{u}$ may be neglected compared to $\mathbf{v}_{c}$ so that eq. (38) attains the well known form of the Navier-Stokes equation

$$
\widehat{\rho} \frac{\partial \mathbf{v}_{\mathrm{c}}}{\partial t}+\widehat{\rho}\left(\mathbf{v}_{c} \cdot \nabla \mathbf{v}_{c}\right)-\mu \Delta \mathbf{v}_{c}+\nabla p=0
$$

where $\mu=\nu \widehat{\rho}$. We have for simplicity also assumed that there is no external force. Our derivation of eq. (39) is esssentially identical with that given by Gebelein [34].

For the objective of the present article the occurrence of the osmotic velocity $\mathbf{u}$ is absolutely vital. Moreover, it is advisable to express the convective velocity $\mathbf{v}_{c}$ by the total velocity $\mathbf{v}=\mathbf{v}_{c}+\mathbf{u}$. Eq. (39) then reads

$$
\frac{\partial}{\partial t}(\mathbf{v}-\mathbf{u})+[(\mathbf{v}+\mathbf{u}) \cdot \nabla(\mathbf{v}-\mathbf{u})]-\nu \Delta(\mathbf{v}-\mathbf{u})=\frac{1}{m_{0}} \mathbf{F}(\mathbf{r}) .
$$

The external force effects an acceleration $\ddot{\sigma}_{f}=\mathbf{F} / m_{0}$ of the $j$-particle and thereby a change of its kinetic energy within $\Delta t$ seconds

$$
\Delta E_{\mathrm{kin} .}^{(j)}=\Delta t \sum_{k=1}^{3} F_{k} \dot{\sigma}_{j k}=\Delta t m_{0} \sum_{k=1}^{3} \ddot{\sigma}_{j k} \dot{\sigma}_{j k}=\Delta t \frac{m_{0}}{2} \frac{d}{d t} \dot{\sigma}_{j}^{2}
$$

On forming the ensemble average and using eqs. (7) and (11)

$$
\dot{\dot{\sigma}}^{2}=\underline{\dot{\sigma}}_{\mathrm{c}}^{2}+\dot{\dot{\sigma}}_{r}^{2} ; \quad \dot{\dot{\sigma}}_{r}^{2}=3 \frac{k_{\mathrm{B}} T}{m_{0}} \longrightarrow \frac{\partial}{\partial t} \dot{\dot{\sigma}}_{r}^{2}=0,
$$

we obtain after time integration

$$
E_{\text {kin. }}=\frac{m_{0}}{2} \mathbf{v}_{\mathrm{c}}^{2} \quad \text { where } \quad \mathbf{v}_{c}=\mathbf{v}_{c}(\mathbf{r}, t)
$$

Thus, the kinetic energy becomes

$$
\epsilon_{\text {kin. }}(\mathbf{r}, t)=\frac{m_{0}}{2} \rho(\mathbf{r}, t)\left[\mathbf{v}_{\mathrm{c}}^{\mathrm{B}}(\mathbf{r}, t)\right]^{2} .
$$

Here we have added the superscript $B$ to characterize $\mathbf{v}_{c}(\mathbf{r}, t)$ as being associated with the Brownian ensemble. 


\section{The motional behavior of a quantum mechanical particle}

As already alluded to in Sect. 2 we subdivide the ensemble of $N$ one-particles systems into a Brownian sub-ensemble "B" where each particle obeys the Langevin equation (6) for a time interval of $\Delta t$ seconds where $\Delta t \gg \tau$, and into an anti-Brownian sub-ensemble "A" where the motion of each particle is governed by

$$
m_{0} \ddot{\sigma}_{j k}-\frac{m_{0}}{\tau} \dot{\sigma}_{j k}=F_{j k}+F_{r j k}^{\mathrm{A}}(t)
$$

In principle, all previous considerations in deriving eq. (40) carry over to the present case where only $\nu$ and - consequently $-\mathbf{u}$ change their sign:

$$
\frac{\partial}{\partial t}(\mathbf{v}+\mathbf{u})+[(\mathbf{v}-\mathbf{u}) \cdot \nabla(\mathbf{v}+\mathbf{u})]+\nu \Delta(\mathbf{v}+\mathbf{u})=\frac{1}{m_{0}} \mathbf{F}(\mathbf{r})
$$

It has to be observed, however, that the Smoluchowski equation (32) must be replaced by

$$
\rho(\mathbf{r}, t-\Delta t) v_{c k}(\mathbf{r}, t-\Delta t)=\int \rho(\mathbf{r}+\underline{\sigma}, t) v_{c k}(\mathbf{r}+\underline{\sigma}, t) P^{\mathrm{A}}(\mathbf{r}+\underline{\sigma},-\underline{\sigma}, t,-\Delta t) d^{3} \sigma,
$$

where $-\sigma$ stands in place of $\sigma$ and $-\Delta t$ in place of $\Delta t$ which amounts to reconstructing $\rho(\mathbf{r}, t-\Delta t) v_{c k}(\mathbf{r}, t-$ $\Delta t)$ from $\rho(\mathbf{r}+\underline{\sigma}, t) v_{c k}(\mathbf{r}+\underline{\sigma}, t)$ by following the scatterings within the time interval of $\Delta t$ seconds backward in time. Since one is now dealing with a system of negative kinematic viscosity $(-1 / \tau$ leads to $-\nu)$ one obtains from the above Smoluchowski equation

$$
\int \sigma_{l} \sigma_{k} P^{\mathrm{A}}(\mathbf{r}+\underline{\sigma},-\underline{\sigma}, t,-\Delta t) d^{3} \sigma=-2 \nu(-\Delta t)=2 \nu \Delta t
$$

which is positive as before. As a matter of course, the mean square shift of a particle can only be positive. That $\nu$ and $\mathbf{u}$ change sign, follows immediately from inspection of eq. (15) and eq. (31).

If one expresses $\mathbf{u}$ in eqs. (40), (43) by $-\nu \nabla \rho / \rho$, one recognizes that there are four terms which scale as $\nu$ and two terms are quadratic in $\nu$. Hence, on forming the arithmatic mean of both equations the terms linear in $\nu$ drop out and those quadratic in $\nu$ remain. We thus arrive at

$$
\underbrace{\frac{\partial}{\partial t} \mathbf{v}+(\mathbf{v} \cdot \nabla) \mathbf{v}}_{=\frac{d}{d t} \mathbf{v}}-(\mathbf{u} \cdot \nabla) \mathbf{u}+\nu \Delta \mathbf{u}=\frac{1}{m_{0}} \mathbf{F}(\mathbf{r}) \text {. }
$$

This differential equation describes the average behavior of the combined A-and B-subsystems, and, as we shall demonstrate in the ensuing Sections, a frictionless particle motion despite the occurrence of the kinematic viscosity. As $\nu$ was given by eq. (15):

$$
\nu=\frac{k_{\mathrm{B}} T \tau}{m_{0}}
$$

one recognizes that the numerator on the rhs has the dimension of an action. On the other hand, that product describes the effect of vacuum fluctuations on the particle under study, no matter what the properties of the particle are, concerning shape, mass and charge. It should therefore be a universal constant of that dimension. Setting

$$
\frac{\hbar}{2}=k_{\mathrm{B}} T \tau \longrightarrow \nu=\frac{\hbar}{2 m_{0}}
$$

turns out to yield - at the non-relativistic level - complete agreement with the experiment, most notably with the energy spectrum of the hydogen atom which has always served as an absolute gauge. Eq. (44) will 
prove to be equivalent to the one-particle Schrödinger equation. However, different from the latter, eq. (44) allows an immediate transition to Newton's second law by letting $\nu=\hbar / 2 m_{0}$ tend to zero, which means absence of vacuum fluctuations. In that case eq. (44) reduces to

$$
\frac{d}{d t} \mathbf{v}=\frac{1}{m_{0}} \mathbf{F} .
$$

In the forward direction of time eq. (43) describes the behavior of an ensemble that collects energy from the vacuum as opposed to the B-ensemble governed by eq. (40) which diffuses energy into the vacuum. The exchange of energy with the vacuum is connected with those terms in the two equations that are linear in $\nu$ and hence drop out on forming the arithmetic mean of the two equations. The latter is done assuming that the respective densities $\rho(\mathbf{r}, t)$ and velocities $\mathbf{v}(\mathbf{r}, t)$ of the two sub-ensembles are equal. (Note: if $\rho(\mathbf{r}, t)$ is the same in both systems, the absolute values of the osmotic velocities agree as well because of $\mathbf{u}=-\nu \nabla \rho / \rho$. The different signs are explicitly taken care of in eq. (43).) But in uncritically forming that average one obscures a crucial assumption that bears directly on the interchange of A- and B-particles. As the two sub-ensembles obey their respective equations eq. (40) and (43), their time evolution is definitely different. If one ensures that $\rho(\mathbf{r}, t)$ and $\mathbf{v}(\mathbf{r}, t)$ of the two sub-ensembles agree at time $t$, this is no longer the case after the elapse of a time $\Delta t \gg \tau$. To guarantee that the two ensembles start again with their respective functions $\rho(\mathbf{r}, t)$ and $\mathbf{v}(\mathbf{r}, t)$ being equal at $t+\Delta t$ one has to exchange particles between the two ensembles, for example, faster ones from $\mathrm{A}$ for slower ones from $\mathrm{B}$ until

$$
\mathbf{v}^{\mathrm{A}}(\mathbf{r}, t+\Delta t)=\mathbf{v}^{\mathrm{B}}(\mathbf{r}, t+\Delta t) .
$$

Since the continuity equation holds for both sub-ensembles:

$$
\dot{\rho}^{\mathrm{A} / \mathrm{B}}=-\operatorname{div}\left(\rho^{\mathrm{A} / \mathrm{B}} \mathbf{v}^{\mathrm{A} / \mathrm{B}}\right)
$$

their densities

$$
\rho^{\mathrm{A} / \mathrm{B}}(\mathbf{r}, t+\Delta t)=\rho^{\mathrm{A} / \mathrm{B}}(\mathbf{r}, t)-\Delta t \operatorname{div}\left(\rho^{\mathrm{A} / \mathrm{B}}(\mathbf{r}, t) \mathbf{v}^{\mathrm{A} / \mathrm{B}}(\mathbf{r}, t)\right)
$$

agree to first order in $\Delta t$ if they and their respective velocities agree at time $t$.

It is exactly the above exchange of particles between the two sub-ensembles which mimics the memory of the vacuum on the particle's previous energy and momentum.

Since $\mathbf{u}$ is given by $-\nu \nabla \rho / \rho$, it is curl-free:

$$
\nabla \times \mathbf{u}(\mathbf{r}, t)=0,
$$

and for this reason we have

$$
(\mathbf{u} \cdot \nabla) \mathbf{u}=-\frac{1}{2} \nabla \mathbf{u}^{2} .
$$

Furthermore $\nu \Delta \mathbf{u}$ can be cast as

$$
\nu \Delta \mathbf{u}(\mathbf{r}, t)=-\nu^{2} \nabla\left[\Delta \ln \left(\rho(\mathbf{r}, t) / \rho_{0}\right)\right]
$$

where $\rho_{0}$ is a constant reference density without physical importance.

We can therefore rewrite eq. (44) in the form

$$
\frac{d}{d t} \mathbf{v}(\mathbf{r}, t)=-\nabla P(\mathbf{r}, t)
$$

where

$$
P(\mathbf{r}, t)=\frac{1}{m_{0}} V(\mathbf{r})-\frac{1}{2} \mathbf{u}^{2}(\mathbf{r}, t)-\nu^{2} \Delta \ln \left(\rho(\mathbf{r}, t) / \rho_{0}\right) .
$$


As has been shown by Helmholtz [35] in connection with treating vortices in ideal fluids, eq. (49) ensures that $\mathbf{v}(\mathbf{r}, t)$ is curl-free except for vortex lines where $\nabla \times \mathbf{v}(\mathbf{r}, t)$ is infinite. However

$$
\oint_{C} \mathbf{v}(\mathbf{r}, t) \neq \infty
$$

for any chosen contour encircling that line. We may therefore set

$$
\mathbf{v}(\mathbf{r}, t)=\frac{\hbar}{m_{0}} \nabla \varphi(\mathbf{r}, t)
$$

for any $\mathbf{r}$ different from points of the vortex line. As the solutions to eq. (44) are curl-free the motion of the particle is frictionless.

\section{Current density and expectation values of momentum, angular momentum and kinetic energy}

We have arrived at the result that the one-particle system under study can uniquely be described by two scalar functions $\rho(\mathbf{r}, t)$ and $\varphi(\mathbf{r}, t)$. These two independent functions may be condensed into a complex-valued scalar function

$$
\psi(\mathbf{r}, t)= \pm \sqrt{\rho(\mathbf{r}, t)} \mathrm{e}^{i \varphi(\mathbf{r}, t)} .
$$

The \pm sign in front of the square root has to be chosen such that $\psi(\mathbf{r}, t)$ remains differentiable at surfaces where $\rho(\mathbf{r}, t)$ vanishes. It turns out that in such a case $\rho$ is time-independent. If $\rho(\mathbf{r})$ varies along a $\xi$ coordinate axis perpendicular to that surface as $\left(\xi-\xi_{0}\right)^{2}$ near the zero $\xi_{0}$ of $\rho(\mathbf{r}), \psi(\mathbf{r})$ would vary as $\left|\xi-\xi_{0}\right|$ and would hence not be differentiable at $\xi_{0}$ except one changes the sign in front of $\sqrt{\rho(\mathbf{r})}$ accordingly.

As $\rho(\mathbf{r}, t)$ and $\mathbf{v}(\mathbf{r}, t)$ will in general be unique (and smooth) functions of $\mathbf{r}$ we require $\psi(\mathbf{r}, t)$ to have the same property. In systems where $V(\mathbf{r})$ is centro- or axi-symmetric, we may introduce spherical or cylindrical coordinates $\mathbf{r}=(r, \vartheta, \phi)$ and $\mathbf{r}=(r, z, \phi)$, respectively, with $r=0$ referring to the center or the axis. Uniqueness of $\psi(\mathbf{r})$ then implies

$$
\varphi(r, \vartheta, \phi+2 \pi)=\varphi(r, \vartheta, \phi)+2 n \pi \quad \text { and } \quad \varphi(r, z, \phi+2 \pi)=\varphi(r, z, \phi)+2 n \pi,
$$

where

$$
n=0,1,2, \ldots
$$

Thus, we have for any contour integral encircling a vortex line

$$
\oint_{\mathrm{c}} \nabla \varphi(\mathbf{r}) \cdot d \mathbf{r}=2 \pi n \text {. }
$$

Insertion of eq. (51) and multiplication by $m_{0}$ yields

$$
\oint_{\mathrm{c}} \mathbf{p}(\mathbf{r}) \cdot d \mathbf{r}=n 2 \pi \hbar \quad \text { where } \quad \mathbf{p}=m_{0} \mathbf{v} .
$$

Obviously, the integral represents the angular momentum of the particle around the vortex line, and eq. (54) is therefore identical with the Bohr-Sommerfeld description for the quantization of angular momentum. In case that $V(\mathbf{r})$ is spherically symmetric, the vortex line is straight and becomes identical with the so-called quantization axis. 
Setting

$$
\psi=|\psi| \mathrm{e}^{i \varphi}
$$

we recognize that

$$
\nabla \psi=\psi\left[i \nabla \varphi+\frac{1}{|\psi|} \nabla|\psi|\right] .
$$

Thus we have

$$
\psi^{*} \nabla \psi=\rho\left[i \nabla \varphi+\frac{1}{|\psi|} \nabla|\psi|\right]
$$

and likewise

$$
\psi \nabla \psi^{*}=\rho\left[-i \nabla \varphi+\frac{1}{|\psi|} \nabla|\psi|\right] .
$$

Subtraction of the latter expression from the former and muliplication by $\hbar / 2 m_{0}$ yields

$$
\mathbf{j}(\mathbf{r}, t)=\frac{\hbar}{2 i m_{0}}\left[\psi^{*} \nabla \psi-\psi \nabla \psi^{*}\right]
$$

where we have used eq. (51) and the definition of the total current density:

$$
\mathbf{j}(\mathbf{r}, t)=\rho(\mathbf{r}, t) \mathbf{v}(\mathbf{r}, t) .
$$

The quantity $m_{0} \mathbf{j}$ represents the momentum density $\mathbf{j}_{p}$ whose real-space integral gives the total momentum of the particle

$$
<\mathbf{p}(t)>=\int_{V} \mathbf{j}_{p}(\mathbf{r}, t) d^{3} r=\frac{1}{2} \int_{V} \psi^{*}(\mathbf{r}, t) \widehat{\mathbf{p}} \psi(\mathbf{r}, t) d^{3} r-c . c .
$$

where $\widehat{\mathbf{p}}$ is short-hand for

$$
\widehat{\mathbf{p}}=-i \hbar \nabla
$$

We have, furthermore, introduced brackets $\langle>$ to make contact to the familiar notation in quantum mechanics. Integration by parts of the second integral in eq. (57) yields the first integral with the opposite sign. Thus we have

$$
<\mathbf{p}(t)>=\int_{V} \psi^{*}(\mathbf{r}, t) \widehat{\mathbf{p}} \psi(\mathbf{r}, t) d^{3} r
$$

which suggests to term $\widehat{\mathbf{p}}$ "momentum operator".

To obtain the simple form of the second integral in eq. (57) in performing the integration by parts, $\psi(\mathbf{r}, t)$ has to vanish sufficiently fast toward the surface of $V$ as that volume goes to infinity. Otherwise it has to fulfill periodic boundary conditions at the six faces of $V$ which must have the form of a parallelepiped.

A free particle of a definite classical momentum is associated with an $\mathbf{r}$-independent ensemble average $m_{0} \mathbf{v}$ because the vacuum fluctuations can - on the average - not lead to a departure from its classical momentum. If follows then from eq. (51)

$$
\varphi(\mathbf{r})=\frac{m_{0}}{\hbar} \int_{\mathbf{r}_{\mathbf{o}}}^{\mathbf{r}} \mathbf{v} \cdot d \mathbf{r}^{\prime}=\frac{m_{0}}{\hbar} \mathbf{v} \cdot \mathbf{r}-\varphi_{0}
$$


where

$$
\varphi_{0}=\frac{m_{0}}{\hbar} \mathbf{v} \cdot \mathbf{r}_{0}
$$

Hence, we have from eq. (53)

$$
\psi(\mathbf{r})=\mathrm{e}^{i \varphi_{0}} \frac{1}{\sqrt{V}} \mathrm{e}^{i \mathbf{k} \cdot \mathbf{r}}
$$

where $\mathbf{k}$ is defined as $\mathbf{k}=\frac{m_{0}}{\hbar} \mathbf{v}$.

In a force-free volume $V$ the vacuum fluctuations can shift the particle trajactory sideways at random, and therefore its probability of position is the same everywhere so that $\rho(\mathbf{r})=1 / V$. As expressly stated in Sect. 2, it would be physically meaningless to interpret eq. (60) as referring to a situation where "the particle is a wave".

The expectation value $<\mathbf{L}>$ of the angular momentum can be obtained in complete analogy to eq. (57) by forming

$$
<\mathbf{L}(t)>=\int_{V}\left(\mathbf{r} \times \mathbf{j}_{p}(\mathbf{r}, t)\right) d^{3} r=\int_{V} \psi^{*}(\mathbf{r}, t)(\mathbf{r} \times \widehat{\mathbf{p}}) \psi(\mathbf{r}, t) d^{3} r .
$$

It is therefore plausible to name

$$
\widehat{\mathbf{L}}=\mathbf{r} \times \widehat{\mathbf{p}}
$$

"angular momentum operator".

We finally consider the kinetic energy density which is given by eq. (41) for the B-ensemble

$$
\epsilon_{\mathrm{kin} .}^{\mathrm{B}}=m_{0} \frac{\rho}{2}\left[\mathbf{v}_{\mathrm{c}}^{\mathrm{B}}\right]^{2}
$$

and accordingly

$$
\epsilon_{\mathrm{kin} .}^{\mathrm{A}}=m_{0} \frac{\rho}{2}\left[\mathbf{v}_{\mathrm{c}}^{\mathrm{A}}\right]^{2}
$$

for the A-ensemble. From eq. (30) we have

$$
\mathbf{v}_{\mathrm{c}}^{\mathrm{B}}=\mathbf{v}-\mathbf{u} \text { and correspondingly for the A-ensemble: } \quad \mathbf{v}_{\mathrm{c}}^{\mathrm{A}}=\mathbf{v}+\mathbf{u}
$$

Thus, the arithmetic mean of the two expressions becomes

$$
\epsilon_{\text {kin. }}=m_{0} \frac{\rho}{2}\left(\mathbf{v}^{2}+\mathbf{u}^{2}\right) .
$$

We may recast eq. (52) as

$$
\ln \left(\psi / \sqrt{\rho_{0}}\right)=\frac{1}{2} \ln \left(\rho / \rho_{0}\right)+i \varphi
$$

multiply this equation by $\hbar / m_{0}$ and form the gradient. Because of eq. (30) and (51) defining $\mathbf{u}$ and $\mathbf{v}$ the result can be written

$$
\frac{\hbar}{m_{0}} \frac{1}{\psi} \nabla \psi=-\mathbf{u}+i \mathbf{v}
$$

The square modulus of this equation, muliplied by $m_{0} \rho / 2$ is obviously identical with the kinetic energy density:

$$
\epsilon_{\text {kin. }}(\mathbf{r}, t)=\frac{\hbar^{2}}{2 m_{0}}|\nabla \psi(\mathbf{r}, t)|^{2}
$$


Hence the kinetic energy is given by

$$
E_{\text {kin. }}=\left\langle T>=\int_{V} \frac{\hbar^{2}}{2 m_{0}} \nabla \psi^{*}(\mathbf{r}, t) \cdot \nabla \psi(\mathbf{r}, t) d^{3} r .\right.
$$

Invoking Green's theorem the integral can be rewritten

$$
\int_{V} \frac{\hbar^{2}}{2 m_{0}} \nabla \psi^{*}(\mathbf{r}, t) \cdot \nabla \psi(\mathbf{r}, t) d^{3} r=\int_{V} \psi^{*}(\mathbf{r}, t)\left[-\frac{\hbar^{2} \nabla^{2}}{2 m_{0}}\right] \psi(\mathbf{r}, t) d^{3} r
$$

and we arrive at

$$
<T>=\int_{V} \psi^{*}(\mathbf{r}, t) \frac{\widehat{\mathbf{p}}^{2}}{2 m_{0}} \psi(\mathbf{r}, t) d^{3} r
$$

which justifies terming

$$
\widehat{\mathbf{T}}=\frac{\widehat{\mathbf{p}}^{2}}{2 m_{0}}=-\frac{\hbar^{2} \nabla^{2}}{2 m_{0}}
$$

the "operator of kinetic energy". It should be recognized, however, that the integrand of eq. (66) will in general not be real-valued any more, as opposed to the integrand in eq. (65) which one obtains primarily. It is, furthermore, worth noticing that the operators $\widehat{\mathbf{p}}, \widehat{\mathbf{L}}$ and $\widehat{\mathbf{T}}$ appear at a stage of the derivation where one has not yet arrived at the Schrödinger equation.

\section{The time-dependent Schrödinger equation}

The crucial eq. (49) may be regarded as the quantum mechanical modification of Newton's second law. We rewrite this equation in the form

$$
\frac{\partial}{\partial t} \mathbf{v}=-\frac{1}{m_{0}} \nabla V-\frac{1}{2} \nabla \mathbf{v}^{2}+\frac{1}{2} \nabla \mathbf{u}^{2}-\frac{\hbar}{2 m_{0}} \Delta \mathbf{u}
$$

The osmotic velocity u was defined via Fick's law by eq. (30) which we cast as

$$
\mathbf{u}=-\frac{\hbar}{2 m_{0}} \nabla \ln \left(\rho / \rho_{0}\right)
$$

Time differentiation yields

$$
\frac{\partial}{\partial t} \mathbf{u}=-\frac{\hbar}{2 m_{0}} \nabla\left(\frac{\partial \rho}{\partial t} / \rho\right)
$$

where $\partial \rho / \partial t$ can be eliminated by using the continuity equation (25). That gives

$$
-\frac{\hbar}{2 m_{0}} \nabla\left(\frac{\partial \rho}{\partial t} / \rho\right)=\frac{\hbar}{2 m_{0}} \nabla \operatorname{div} \mathbf{v}-\nabla\left[\mathbf{v} \cdot\left(-\frac{\hbar}{2 m_{0}} \frac{1}{\rho} \nabla \rho\right)\right] .
$$

Because of eq. (69) the bracketed term on the rhs may be replaced by $\mathbf{v} \cdot \mathbf{u}$. As $\mathbf{v}$ is curl-free, the first term on the rhs is just $\hbar / 2 m_{0} \Delta \mathbf{v}$, and hence one gets as an equivalent of eq. (69)

$$
\frac{\partial}{\partial t} \mathbf{u}=\frac{\hbar}{2 m_{0}} \Delta \mathbf{v}-\nabla(\mathbf{u} \cdot \mathbf{v})
$$


If we multiply eq. (68) by the imaginary unit $i$ and subtract eq. (71) we obtain

$$
\frac{\partial}{\partial t}(-\mathbf{u}+i \mathbf{v})=-\frac{i}{m_{0}} \nabla V-\frac{i}{2} \nabla \mathbf{v}^{2}+\frac{i}{2} \nabla \mathbf{u}^{2}-i \frac{\hbar}{2 m_{0}} \Delta \mathbf{u}-\frac{\hbar}{2 m_{0}} \Delta \mathbf{v}+\nabla(\mathbf{u} \cdot \mathbf{v})
$$

or after reordering on the right

$$
\frac{\partial}{\partial t}(-\mathbf{u}+i \mathbf{v})=\frac{i}{2} \nabla(-\mathbf{u}+i \mathbf{v})^{2}+\frac{i \hbar}{2 m_{0}} \nabla[\nabla(-\mathbf{u}+i \mathbf{v})]-\frac{i}{m_{0}} \nabla V .
$$

From eq. (63) we have

$$
\frac{\hbar}{m_{0}} \nabla\left(\ln \psi / \sqrt{\rho_{0}}\right)=-\mathbf{u}+i \mathbf{v}
$$

which we insert into eq. (72). Interchanging the operators $\partial / \partial t$ and $\nabla$ on the lhs one gets

$$
\nabla\left(\frac{\hbar}{m_{0}} \frac{1}{\psi} \frac{\partial \psi}{\partial t}\right)=\nabla\left[\frac{i}{2} \frac{\hbar^{2}}{m_{0}^{2}}\left\{\left(\frac{1}{\psi} \nabla \psi\right)^{2}+\nabla \cdot\left(\frac{1}{\psi} \nabla \psi\right)\right\}-\frac{i}{m_{0}} V\right] .
$$

If the gradient of two functions are equal then the functions can only differ by a time-dependent real-space independent function which we denote by $\beta(t)$. Hence, we have after division by $i$ :

$$
-i \frac{\hbar}{m_{0}} \frac{1}{\psi} \frac{\partial \psi}{\partial t}=\frac{1}{2} \frac{\hbar^{2}}{m_{0}^{2}}\left[\left(\frac{1}{\psi} \nabla \psi\right)^{2}+\nabla \cdot\left(\frac{1}{\psi} \nabla \psi\right)\right]-\frac{i}{m_{0}} V-i \beta(t) .
$$

We observe that

$$
\nabla \cdot\left(\frac{1}{\psi} \nabla \psi\right)=-\left(\frac{1}{\psi} \nabla \psi\right)^{2}+\frac{1}{\psi} \nabla^{2} \psi
$$

and multiply eq. (74) by $-m_{0} \psi$, which gives

$$
i \hbar \frac{\partial \psi}{\partial t}=-\frac{\hbar^{2} \nabla^{2}}{2 m_{0}} \psi+V \psi+\gamma(t) \psi
$$

where

$$
\gamma(t)=i m_{0} \beta(t)
$$

If we now replace $\psi(\mathbf{r}, t)$ by $\widehat{\psi}(\mathbf{r}, t)$ defined through

$$
\psi(\mathbf{r}, t)=\widehat{\psi}(\mathbf{r}, t) \exp \left[-\frac{i}{\hbar} \int_{t_{0}}^{t} \gamma\left(t^{\prime}\right) d t^{\prime}\right]
$$

eq. (75) turns into a differential equation for $\widehat{\psi}(\mathbf{r}, t)$ :

$$
i \hbar \frac{\partial \widehat{\psi}(\mathbf{r}, t)}{\partial t}=\left[\frac{\widehat{\mathbf{p}}^{2}}{2 m_{0}}+V(\mathbf{r})\right] \widehat{\psi}(\mathbf{r}, t),
$$

where we have used eq. (67) to express $-\hbar^{2} \nabla^{2} / 2 m_{0}$. The total energy of the particle is given by

$$
E=E_{\text {kin. }}+\int \rho(\mathbf{r}, t) V(\mathbf{r}) d^{3} r .
$$


Using eq. (66) we may rewrite this expression

$$
E=\int \psi^{*}(\mathbf{r}, t)\left[\frac{\widehat{\mathbf{p}}^{2}}{2 m_{0}}+V(\mathbf{r})\right] \psi(\mathbf{r}, t) d^{3} r=\int \psi^{*}(\mathbf{r}, t) \widehat{\mathbf{H}} \psi(\mathbf{r}, t) d^{3} r
$$

We are therefore justified in terming

$$
\widehat{H}(\mathbf{r})=\frac{\widehat{\mathbf{p}}^{2}}{2 m_{0}}+V(\mathbf{r})
$$

"total energy operator" or "Hamilton operator".

Eq. (77) represents the time-dependent Schrödinger equation. The functions $\psi(\mathbf{r}, t)$ and $\widehat{\psi}(\mathbf{r}, t)$ differ by a phase factor which is of no physical importance because the only relevant information on the state of the system is connected with

$$
\rho(\mathbf{r}, t)=\psi^{*}(\mathbf{r}, t) \psi(\mathbf{r}, t)=\widehat{\psi}^{*}(\mathbf{r}, t) \widehat{\psi}(\mathbf{r}, t) \quad \text { and } \quad \mathbf{j}(\mathbf{r}, t)=\rho(\mathbf{r}, t) \nabla \varphi(\mathbf{r}, t) .
$$

Neither expression depends on this phase factor. We may therefore justifiably ignore the distinction between $\psi(\mathbf{r}, t)$ and $\widehat{\psi}(\mathbf{r}, t)$. It is worth noticing that the occurrence of the first order time-derivative in the Schrödinger equation (77) can be traced back to $\partial \mathbf{v} / \partial t$ in Newton's modified second law eq. (68) and to $\partial \mathbf{u} / \partial t$ in the equation of diffusion (70).

\section{The Schrödinger equation in the presence of an external electromagnetic field}

Reexamining the various steps in the derivation of the Schrödinger equation (77) one notices that all considerations remain unaffected if one allows the external force to be time-dependent: $\mathbf{F}=\nabla V(\mathbf{r}, t)$. As a matter of principle, Newton's modified second law in the form of eq. (44) holds also in the presence of a magnetic field $\mathbf{B}(\mathbf{r}, t)$, except that $\mathbf{F}$ is now given by

$$
\mathbf{F}=e \hat{\mathbf{E}}(\mathbf{r}, t)+e \mathbf{v}(\mathbf{r}, t) \times \mathbf{B}(\mathbf{r}, t) .
$$

Let $\mathbf{A}(\mathbf{r}, t)$ be the vector potential associated with $\mathbf{B}(\mathbf{r}, t)$ :

$$
\mathbf{B}(\mathbf{r}, t)=\nabla \times \mathbf{A}(\mathbf{r}, t) .
$$

We recall the property of the contour integral

$$
\oint_{C} \mathbf{p}(\mathbf{r}, t) \cdot d \mathbf{r}=0
$$

if the integration path does not encircle a vortex line and if there is no magnetic field.

By switching on a magnetic field one induces a circular voltage along the contour:

$$
\oint_{C} \hat{\mathbf{E}}_{\text {ind. }}\left(\mathbf{r}^{\prime}, t\right) \cdot d \mathbf{r}^{\prime}=-\frac{\partial}{\partial t} \int_{\mathrm{A}} \mathbf{B}\left(\mathbf{r}^{\prime}, t\right) \cdot d^{2} \mathbf{r}^{\prime}
$$

where $A$ is the area circumscribed by the contour. Using Stoke's theorem and muliplying by $e$ we may rewrite this equation:

$$
\oint_{C} e \hat{\mathbf{E}}_{\mathrm{ind} .}\left(\mathbf{r}^{\prime}, t\right) \cdot d \mathbf{r}^{\prime}=-\frac{\partial}{\partial t} \oint_{C} e \mathbf{A}\left(\mathbf{r}^{\prime}, t\right) \cdot d \mathbf{r}^{\prime}
$$


The integrand on the lhs describes an additional force $\mathbf{F}_{\text {ind. }}\left(\mathbf{r}^{\prime}, t\right)$ acting on the charged particle and effecting a change of the particle's momentum:

$$
\oint_{C} \dot{\mathbf{p}}\left(\mathbf{r}^{\prime}, t^{\prime}\right) \cdot d \mathbf{r}^{\prime}=\oint_{C} e \hat{\mathbf{E}}_{\text {ind. }}\left(\mathbf{r}^{\prime}, t^{\prime}\right) \cdot d \mathbf{r}^{\prime}=-\frac{\partial}{\partial t^{\prime}} \oint_{C} e \mathbf{A}\left(\mathbf{r}^{\prime}, t^{\prime}\right) \cdot d \mathbf{r}^{\prime} .
$$

We perform a time integration of this equation from $t^{\prime}=t_{0}$ to $t$ and observe that $\mathbf{A}\left(\mathbf{r}^{\prime}, t_{0}\right) \equiv 0$. The result may be cast as

$$
\oint_{C} \mathbf{p}\left(\mathbf{r}^{\prime}, t\right) \cdot d \mathbf{r}^{\prime}=-\oint_{C} e \mathbf{A}\left(\mathbf{r}^{\prime}, t\right) \cdot d \mathbf{r}^{\prime}
$$

or alternatively

$$
\oint\left[\mathbf{v}\left(\mathbf{r}^{\prime}, t\right)+\frac{e}{m_{0}} \mathbf{A}\left(\mathbf{r}^{\prime}, t\right)\right] \cdot d \mathbf{r}^{\prime}=0 \quad \text { equivalent to } \quad \nabla \times\left[\mathbf{v}(\mathbf{r}, t)+\frac{e}{m_{0}} \mathbf{A}(\mathbf{r}, t)\right] \equiv 0 .
$$

Hence, $\mathbf{v}+\frac{e}{m_{0}} \mathbf{A}$ must be expressible as a gradient of a scalar function which we write $\left(\hbar / m_{0}\right) \varphi(\mathbf{r}, t)$ :

$$
\mathbf{v}(\mathbf{r}, t)+\frac{e}{m_{0}} \mathbf{A}(\mathbf{r}, t)=\frac{\hbar}{m_{0}} \nabla \varphi(\mathbf{r}, t) .
$$

This relation replaces the previous eq. (51) for zero magnetic field. By contrast, eq. (70) for the osmotic velocity remains unaffected by the presence of the magnetic field. As a consequence of the fact that $\mathbf{v}(\mathbf{r}, t)$ is no longer curl-free, the expression $(\mathbf{v} \cdot \nabla) \mathbf{v}$ in eq. (44) becomes now slightly more complicated:

$$
(\mathbf{v} \cdot \nabla) \mathbf{v}=\frac{1}{2} \nabla \mathbf{v}^{2}-\mathbf{v} \times(\nabla \times \mathbf{v})=\frac{1}{2} \nabla \mathbf{v}^{2}+\frac{e}{m_{0}} \mathbf{v} \times(\nabla \times \mathbf{A})
$$

which, on substituting $\nabla \times \mathbf{A}$ by $\mathbf{B}$, may be recast as

$$
(\mathbf{v} \cdot \nabla) \mathbf{v}=\frac{1}{2} \nabla \mathbf{v}^{2}+\frac{e}{m_{0}} \mathbf{v} \times \mathbf{B} .
$$

If this is inserted into eq. (44) where $\mathbf{F}$ is given by eq. (79), the Lorentz force $e \mathbf{v} \times \mathbf{B}$ drops out, and we obtain

$$
\frac{\partial}{\partial t}\left(\mathbf{v}+\frac{e}{m_{0}} \mathbf{A}\right)=-\frac{1}{2} \nabla V-\frac{1}{2} \nabla \mathbf{v}^{2}+\frac{1}{2} \nabla \mathbf{u}^{2}-\frac{\hbar}{2 m_{0}} \Delta \mathbf{u}
$$

where $V=e \hat{V}$. On multiplying eq. (83) by $i$ and subtracting the unaffected eq. (71) we obtain in complete analogy to eq. (72)

$$
\frac{\partial}{\partial t}\left[-\mathbf{u}+i\left(\mathbf{v}+\frac{e}{m_{0}} \mathbf{A}\right)\right]=\frac{i}{2} \nabla(-\mathbf{u}+i \mathbf{v})^{2}+\frac{i \hbar}{2 m_{0}} \nabla[\nabla(-\mathbf{u}+i \mathbf{v})]-\frac{i}{m_{0}} \nabla V .
$$

Since $\mathbf{v}$ is no longer $\propto \nabla \varphi$ but has to expressed with the aid of eq. (82), we have instead of eq. (73)

$$
\frac{\hbar}{m_{0}} \nabla\left(\ln \psi / \sqrt{\rho_{0}}\right)=-\mathbf{u}+i\left(\mathbf{v}+\frac{e}{m_{0}} \mathbf{A}\right) .
$$

The lhs of eq. (84) may hence be expressed by using

$$
\frac{\partial}{\partial t} \frac{\hbar}{m_{0}} \nabla\left(\ln \psi / \sqrt{\rho_{0}}\right)=\nabla\left(\frac{1}{m_{0} \psi} \hbar \frac{\partial}{\partial t} \psi\right) .
$$


In rearranging the rhs we observe that

$$
-\mathbf{u}+i \mathbf{v}=\frac{\hbar}{m_{0}} \nabla\left(\ln \psi / \sqrt{\rho_{0}}\right)-i \frac{e}{m_{0}} \mathbf{A}
$$

and obtain for the first term on the rhs of eq. (84)

$$
\frac{i}{2} \nabla(-\mathbf{u}+i \mathbf{v})^{2}=\frac{1}{2} \frac{\hbar^{2}}{m_{0}^{2}} \nabla\left[\nabla \ln \left(\psi / \sqrt{\rho_{0}}\right)\right]^{2}-i \frac{\hbar}{m_{0}} \frac{e}{m_{0}} \nabla\left[\mathbf{A} \cdot \nabla \ln \left(\psi / \sqrt{\rho_{0}}\right)\right]-\frac{1}{2} \nabla\left(\frac{e}{m_{0}} \mathbf{A}\right)^{2} .
$$

Using

$$
\nabla\left[\nabla \ln \left(\psi / \sqrt{\rho_{0}}\right)\right]=\frac{1}{\psi} \Delta \psi-\left[\nabla \ln \left(\psi / \sqrt{\rho_{0}}\right)\right]^{2}
$$

we can cast the second term on the rhs of eq. (84)

$$
\frac{\hbar}{2 m_{0}} \nabla[\nabla(-\mathbf{u}+i \mathbf{v})]=-\frac{1}{2} \frac{\hbar^{2}}{m_{0}^{2}} \nabla\left[\nabla \ln \left(\psi / \sqrt{\rho_{0}}\right)\right]^{2}+\nabla\left[\frac{1}{\psi}\left(\frac{1}{2} \frac{\hbar^{2}}{m_{0}^{2}} \Delta \psi-\frac{\hbar}{2 m_{0}} \frac{e}{m_{0}} \psi \nabla \cdot \mathbf{A}\right)\right] .
$$

We thus get

$$
\begin{aligned}
& \frac{i}{2} \nabla(-\mathbf{u}+i \mathbf{v})^{2}+\frac{i \hbar}{2 m_{0}} \nabla[\nabla(-\mathbf{u}+i \mathbf{v})] \\
& =-i \nabla\left[\frac{1}{m_{0} \psi}\left(-\frac{\hbar^{2}}{2 m_{0}} \Delta \psi+i \frac{\hbar}{2} \frac{e}{m_{0}} \nabla \cdot \mathbf{A}+i \hbar \frac{e}{m_{0}} \mathbf{A} \cdot \nabla+\frac{1}{2 m_{0}}(e \mathbf{A})^{2}\right) \psi\right]
\end{aligned}
$$

which on introducing $\widehat{\mathbf{p}}$, as defined by eq. (58), may be compactified in the form

$$
\frac{i}{2} \nabla(-\mathbf{u}+i \mathbf{v})^{2}+\frac{i \hbar}{2 m_{0}} \nabla[\nabla(-\mathbf{u}+i \mathbf{v})]=-i \nabla\left[\frac{1}{m_{0} \psi} \frac{(\widehat{\mathbf{p}}-e \mathbf{A})^{2}}{2 m_{0}} \psi\right] .
$$

If this inserted into eq. (84) and $\partial / \partial t\left[-\mathbf{u}+i\left(\mathbf{v}+\left(e / m_{0}\right) \mathbf{A}\right)\right]$ substituted by using eqs. (85) and eqs. (86) we obtain

$$
\left[\frac{\widehat{\mathbf{P}}^{2}}{2 m_{0}}+V(\mathbf{r}, t)\right] \psi(\mathbf{r}, t)=i \hbar \frac{\partial}{\partial t} \psi(\mathbf{r}, t)
$$

where

$$
\widehat{\mathbf{P}}=\widehat{\mathbf{p}}-e \mathbf{A}(\mathbf{r}, t)
$$

which represents the time-dependent Schrödinger equation in the presence of an electromagnetic field.

Newton's modified law (44), which has been the starting point of our derivation, contains via eq. (79) explicitly the magnetic field in terms of the Lorentz force $e \mathbf{v} \times \mathbf{B}$. From this point of view it is very surprising that this equation of motion transforms into a time-dependent Schrödinger equation where $\mathbf{B}(\mathbf{r}, t)$ does no longer appear, but $\mathbf{A}(\mathbf{r}, t)$ instead. This gives rise to unexpected phenomena, most notably to the so-called Aharonov-Bohm effect [36]. The puzzling role played by the vector potential in the quantum mechanical motion of particles is strikingly illustrated by a two-slit experiment where each beam, associated with the pertinent slit, runs through a region where $\mathbf{B}(\mathbf{r})=0$ but $\mathbf{A}(\mathbf{r}) \neq 0$. This is achieved by placing a sufficiently long solenoid between the beams which creates a magnetic field only inside the solenoid. Hence, there can be no magnetic force acting on the beams. Nevertheless, when the two beams merge behind the solenoid, they display an interference pattern which shifts as $\mathbf{A}$ increases. This was most convincingly demonstrated 
by Möllenstedt and Bayh [37]. An earlier experiment by Chambers [38] who used an iron whisker instead of a solenoid, had already revealed the presence of interference fringes but could not demonstrate the dependence on the magnitude of $\mathbf{A}$.

A vital point in our derivation was the occurrence of a circular electric field $\hat{\mathbf{E}}_{\text {ind. }}(\mathbf{r}, t)$ within some time interval during which the magnetic field builds up. All charged elementary particles in the region of space where $\hat{\mathbf{E}}_{\text {ind. }}(\mathbf{r}, t) \neq 0$ are affected in their motional state as $\hat{\mathbf{E}}_{\text {ind }}$ imparts a momentum on them within that time interval. Since the embedding vacuum is frictionless, this information cannot get lost. We can demonstrate this by a thought experiment where one places an electron in a toroidal potential so that it can only move within a torus and set up a stationary circular current. For simplicity we assume that current to be equal to zero in the beginning. The torus encircles a sufficiently long solenoid which ensures that the magnetic field is completely confined the interior of the solenoid. We consider two of such set-ups: one that contains an electron, the other one is still empty. If one now turns on the magnetic field and waits until $\mathbf{B}(\mathbf{r}, t)$ has become stationary in both solenoids, the electron in the first torus has picked up an angular momentum

$$
\oint_{C} \mathbf{p}(\mathbf{r}) \cdot d \mathbf{r}=-\oint_{C} e \mathbf{A}(\mathbf{r}) \cdot d \mathbf{r} .
$$

We now slowly feed the other electron into the second torus such that it would not set up any current if $\mathbf{A}(\mathbf{r})$ were zero. However, in the spirit of our derivation it must attain a state with the same angular momentum as the first electron, although it does not experience any electric field $\hat{\mathbf{E}}_{\text {ind. }}$. But the energy fluctuations in the embedding vacuum are such that the particle is driven into that state. This is remotely similar to a particle that has been placed into a harmonic oscillator potential at a position where the potential is lowest. The vacuum fluctuations force the particle into a state where its average energy is $\frac{1}{2} \hbar \omega_{0}$.

If the vacuum would not transfer the angular momentum $\propto|\mathbf{A}|$, one would run into an inconsistency if the magnetic field would be turned off. Now an electric field $\hat{\mathbf{E}}_{\text {ind. }}$. would act on the second electron and impart an angular momentum on it. One would hence end up with a situation where an electron of primarily zero kinetic energy attains a state of finite angular momentum in a space that is definitely field-free and where another electron in an identical torus would have zero angular momentum in a state of zero energy.

\section{Objections raised against Nelson's theory}

We confine ourselves here to only one article by Mielnik and Tengstrand [39] which presents some apparently serious reservations against Nelson's theory. Objections made by other authors over the years revolve around the same or similar questions.

Mielnik and Tengstrand state that "the effects observed in quantum physics cannot be explained by assuming an interaction between many trajectories of different mass points." As we have emphasized in our derivation, it is absolutely crucial that particles of the A- and B-system have to be interchanged after each time step. This might look like an interaction between the particles of different trajectories, but it is only a trick to mimic the reversibility in the scattering of the particle under study. If there were a formalism available that would allow one to directly describe reversible scattering without the detour via ensemble subdivision and particle interchange, each particle would just follow one trajectory. Reversible scattering represents manifestly a non-classical phenomenon, and hence certain features lack comparability with irreversible scattering as with the Brownian motion of a particle. But still, the notion of particle trajectories and the ensemble-referenced quantities $\rho(\mathbf{r}, t)$ and $\mathbf{v}(\mathbf{r}, t)$ are common elements of these different types of stochastic particle motion.

The fundamental equation of motion (44) as a modification of Newton's second law results from an input/output balance of the momentum flux density for a single cube $\Delta^{3} r_{\nu}$. This is in complete analogy to the derivation of Euler's equation in hydrodynamics. Solutions to the latter are, for example, unattenuated waves that fill the entire space absorbed by the fluid under study. A portion of the fluid that occupies the cube 
$\Delta^{3} r_{\nu}$ and undergoes changes of its density and velocity in that cube, feels indirectly the respective changes in other cubes regardless how distant they are. Because of the removal of dissipative effects, solutions to our eq. (44) have therefore analogous properties. Mielnik and Tengstrand discuss a hypothetical twoslit experiment where one has placed a thin wall between the two beams which separates them over a long distance. The authors claim that Nelson's theory would be unable to explain the occurrence of an interference of the two beams when they merge again at the end of the separating wall. In the light of the foregoing consideration this is simply incorrect.

A seemingly disastrous objection of the authors concerns excited states. As is obvious from our fundamental equation (44) which contains $\partial / \partial t$ as a clear indication of its origin from Newton's second law, this equation describes in general a time-dependent one-particle system. In other words, it will in general describe a non-equilibrium state, distinctly in contrast to what Mielnik and Tengstrand claim. If a theory yields a coherent derivation of the time-dependent Schrödinger equation, it is exceedingly puzzling to read statements like that:"The properties of non-equilibrium states in Nelson's theory are left somewhat obscure". However, as regards excited pure states the authors touch upon a serious problem: an excited pure state will in general possess one or several nodal surfaces where the diffusive current density $\mathbf{j}_{\mathrm{d}}(\mathbf{r})=-\left(\hbar / m_{0}\right) \nabla \rho(\mathbf{r})$ vanishes. Hence, if the particle moves in a spatial region beyond such a surface it would never be able to cross that surface and reach the space on the other side of the surface, which seems implausible and is unphysical in the end. Yet, the preparation of a pure state, whose energy is sharply defined, is impossible in practice, because it would require an infinite preparation time. All experiments that are aimed at excitations into well defined states are actually dealing with a linear combination of pure states (multiplied by their time-dependent exponential function). This linear combination solves the time-dependent Schrödinger equation, but it yields a density that does not possess surfaces any more where the density vanishes. A "pure state" is in practice always a linear combination of this kind where one term has particularly large weight. The true world of excited states invalidates the objection of Mielnik and Tengstrand.

\section{The issue of hidden variables}

In the spirit of J. v. Neumann's proof [40] on the non-existence of hidden variables in quantum mechanics Nelson's theory has to be regarded as a hidden variable theory. The real-space coordinates of the individual particles defining the fundamental ensemble represent "hidden variables" because they do not appear any more once we have arrived at the functions $\rho(\mathbf{r}, t), \mathbf{u}(\mathbf{r}, t)$ and $\mathbf{v}(\mathbf{r}, t)$ and the partial differential equations (68) and (70).This carries over to the time-dependent Schrödinger equation which, together with $\psi(\mathbf{r}, t)= \pm \sqrt{\rho(\mathbf{r}, t)} \exp [i \varphi(\mathbf{r}, t)]$, only compactifies these pieces of information and their differential equations.

It has firmly been believed for 34 years that v. Neumann's fundamental book [40] on the foundation of quantum mechanics contained a solid mathematical proof that there can be no hidden variables theory in quantum mechanics. That there was an error in v. Neumann's interpretation of his proof was shortly later analyzed in a paper by Grete Hermann [43]. However, the article remained unnoticed. In 1966 John Bell rediscovered that $v$. Neumann's proof contained serious flaws, a fact that he later summarized in an interview by stating:"The proof of v. Neumann is not merely false but foolish!" Bell himself proved two no-hidden variables theorems [41,42] which, however, do not call theories of the Bohm- or Nelson-type into question. We therefore wish to leave it at that and take a remark by Mermin [44] (in reference to John Bell) as indirectly giving support to Nelson's approach :"None of the no-hidden variables theorems persuaded him that hidden variables were impossible."

\section{The time-dependent $N$-particle Schrödinger equation}

The conclusions we went through in deriving the one-particle Schrödinger equation carry over to the $\mathrm{N}$ particle case point by point provided that the particles interact via two-body forces. One only has to substitute 
the 3-dimensional configuration space of the pertinent point particle by a $3 \mathrm{~N}$-dimensional space where $N$ particles appear again as one point. In analogy to $\rho(\mathbf{r}, t) d^{3} r$ we now have

$$
\rho\left(\mathbf{r}_{1}, \mathbf{r}_{2}, \ldots \mathbf{r}_{N}, t\right) d^{3} r_{1} d^{3} r_{2} \ldots d^{3} r_{N}
$$

which is the probability of finding at time $t$ particle number 1 at $\mathbf{r}_{1}$, particle number 2 at $\mathbf{r}_{2}$ and so on. Abbreviating

$$
\mathbf{r}^{N}=\left(\mathbf{r}_{1}, \mathbf{r}_{2}, \ldots \mathbf{r}_{N}\right)=\sum_{j=1}^{N} \sum_{k=1}^{3} x_{j k} \mathbf{e}_{j k} \quad \text { where } \quad j=1,2, \ldots N \quad \text { and } \quad k=1,2,3
$$

with $x_{j k}$ denoting cartesian coordinates associated with orthogonal unit vectors $\mathbf{e}_{j k}$. The quantities $\nabla^{N}$, $\mathbf{u}^{N}$ and $\mathbf{v}^{N}$ are defined analogously.

The scalar function $\phi\left(\mathbf{r}^{N}, t\right)$ stands now in place of $\varphi(\mathbf{r}, t)$, and hence

$$
\mathbf{v}^{N}\left(\mathbf{r}^{N}, t\right)=\frac{\hbar}{m_{0}} \nabla^{N} \phi\left(\mathbf{r}^{N}, t\right)
$$

Furthermore we have

$$
\mathbf{u}^{N}\left(\mathbf{r}^{N}, t\right)=-\frac{\hbar}{2 m_{0}} \nabla^{N} \ln \left[\rho\left(\mathbf{r}^{N}, t\right) / \rho_{0}\right]
$$

The external generalized force $\mathbf{F}_{\text {ext. }}^{N}\left(\mathbf{r}^{N}, t\right)$ that acts on the system may be written

$$
\mathbf{F}_{\text {ext. }}^{N}\left(\mathbf{r}^{N}, t\right)=\sum_{j=1}^{N} \sum_{k=1}^{3} F_{k}^{\text {ext. }}\left(\mathbf{r}_{j}, t\right) \mathbf{e}_{j k}
$$

where

$$
F_{k}^{\text {ext. }}\left(\mathbf{r}_{j}, t\right)=-\frac{\partial}{\partial x_{j k}} V_{\text {ext. }}\left(\mathbf{r}_{j}, t\right)
$$

with $V_{\text {ext. }}(\mathbf{r}, t)$ denoting the external potential. Hence $\mathbf{F}_{\text {ext. }}^{N}$ may alternatively be written

$$
\mathbf{F}_{\text {ext. }}^{N}\left(\mathbf{r}^{N}, t\right)=-\nabla^{N} \widehat{V}_{\text {ext. }}\left(\mathbf{r}_{1}, \mathbf{r}_{2}, \ldots \mathbf{r}_{N}, t\right)
$$

where

$$
\widehat{V}_{\text {ext. }}\left(\mathbf{r}_{1}, \mathbf{r}_{2}, \ldots \mathbf{r}_{N}, t\right)=\sum_{j=1}^{N} V_{\text {ext. }}\left(\mathbf{r}_{j}, t\right)
$$

The force that acts on the $j$-th particle due to pair-interaction is given by

$$
F_{j k}^{\text {inter }}\left(\mathbf{r}_{j}\right)=-\frac{\partial}{\partial x_{j k}} \sum_{\substack{i=1 \\ i \neq j}}^{N} V\left(\left|\mathbf{r}_{j}-\mathbf{r}_{i}\right|\right)
$$

with $V\left(\left|\mathbf{r}_{j}-\mathbf{r}_{i}\right|\right)$ denoting the interaction potential. The total generalized force $\mathbf{F}^{N}\left(\mathbf{r}^{N}, t\right)$ may therefore be cast as

$$
\mathbf{F}^{N}\left(\mathbf{r}^{N}, t\right)=-\nabla^{N} \widehat{V}\left(\mathbf{r}_{1}, \mathbf{r}_{2}, \ldots \mathbf{r}_{N}, t\right)
$$


where

$$
\widehat{V}\left(\mathbf{r}_{1}, \mathbf{r}_{2}, \ldots \mathbf{r}_{N}, t\right)=\sum_{j=1}^{N} V_{\text {ext. }}\left(\mathbf{r}_{j}, t\right)+\frac{1}{2} \sum_{j=1}^{N} \sum_{\substack{i=1 \\ i \neq j}}^{N} V\left(\left|\mathbf{r}_{j}-\mathbf{r}_{i}\right|\right) .
$$

Newton's modified second law in the form of eq. (68) now reads

$$
\frac{\partial}{\partial t} \mathbf{v}^{N}=-\frac{1}{m_{0}} \nabla^{N} \widehat{V}-\frac{1}{2} \nabla^{N}\left(\mathbf{v}^{N}\right)^{2}+\frac{1}{2} \nabla^{N}\left(\mathbf{u}^{N}\right)^{2}-\frac{\hbar}{2 m_{0}}\left(\nabla^{N}\right)^{2} \mathbf{u}^{N} .
$$

Likewise one obtains the analogue to eq. (70).

Again we form a complex-valued function $\Psi\left(\mathbf{r}_{1}, \mathbf{r}_{2}, \ldots \mathbf{r}_{N}, t\right)$ from the two scalar functions $\rho\left(\mathbf{r}^{N}, t\right)$ and $\phi\left(\mathbf{r}^{N}, t\right)$ according to

$$
\Psi\left(\mathbf{r}_{1}, \mathbf{r}_{2}, \ldots \mathbf{r}_{N}, t\right)= \pm \sqrt{\rho\left(\mathbf{r}_{1}, \mathbf{r}_{2}, \ldots \mathbf{r}_{N}, t\right)} \exp \left[i \phi\left(\left(\mathbf{r}_{1}, \mathbf{r}_{2}, \ldots \mathbf{r}_{N}, t\right)\right)\right]
$$

Starting, as before, from the equations for $\frac{\partial \mathbf{v}^{N}}{\partial t}$ and $\frac{\partial \mathbf{u}^{N}}{\partial t}$, and going through completely analogous manipulations we arrive at the time-dependent $N$-particle Schrödinger equation

$$
\left[\widehat{H}_{0}+\frac{1}{2} \sum_{\substack{i, j \\ i \neq j}} V\left(\left|\mathbf{r}_{j}-\mathbf{r}_{\mathbf{i}}\right|\right)\right] \Psi\left(\mathbf{r}_{1}, \mathbf{r}_{2}, \ldots \mathbf{r}_{N}, t\right)=i \hbar \frac{\partial}{\partial t} \Psi\left(\mathbf{r}_{1}, \mathbf{r}_{2}, \ldots \mathbf{r}_{N}, t\right)
$$

where

$$
\widehat{H}_{0}=\sum_{j=1}^{N}\left[\frac{\widehat{\mathbf{p}}_{j}^{2}}{2 m_{0}}+V_{\text {ext. }}\left(\mathbf{r}_{j}, t\right)\right]
$$

which can be generalized to include magnetic fields. In that case $\widehat{\mathbf{P}}_{j}=\widehat{\mathbf{p}}_{j}-e \mathbf{A}\left(\mathbf{r}_{j}, t\right)$ stands in place of $\widehat{\mathbf{p}}_{j}$.

There is an important point that deserves particular attention. If an $N$-electron system is in a state that carries electric current, $\mathbf{A}\left(\mathbf{r}_{j}\right)$ contains a contribution

$$
\mathbf{A}^{\prime}\left(\mathbf{r}_{j}\right) \propto \int \frac{\mathbf{j}_{e}\left(\mathbf{r}^{\prime}\right)}{\left|\mathbf{r}_{j}-\mathbf{r}^{\prime}\right|} d^{3} r^{\prime}
$$

where $\mathbf{j}_{e}$ is the current density given by

$$
\begin{aligned}
\mathbf{j}_{e}(\mathbf{r})= & \frac{e \hbar}{2 i m_{0}} N \int\left[\Psi^{*}\left(\mathbf{r}, \mathbf{r}_{2}, \ldots \mathbf{r}_{N}\right) \nabla \Psi\left(\mathbf{r}, \mathbf{r}_{2}, \ldots \mathbf{r}_{N}-c . c .\right] d^{3} r_{2} d^{3} r_{3} \ldots d^{3} r_{N}\right. \\
& +\frac{e^{2}}{m_{0}} N \int\left|\Psi\left(\mathbf{r}, \mathbf{r}_{2}, \ldots \mathbf{r}_{N}\right)\right|^{2} \mathbf{A}(\mathbf{r}) d^{3} r_{2} d^{3} r_{3} \ldots d^{3} r_{N}
\end{aligned}
$$

which means that $\widehat{\mathbf{P}}_{j}$ is now state-dependent, thereby invalidating the superposition principle for states that are solutions to the associated Schrödinger equation. This calls alternative foundations of quantum mechanics into question that are fundamentally tied to the superposition principle.

This article is based on a manuscript by Jutta Aschenbach: "Staatsexamensarbeit" (a required academic thesis for the secondary school teaching physics), Technische Universität Clausthal, Germany (1978)).

\section{References}

[1] J. Bell, Phys. World 38 (August), 33 (1990).

[2] E. Nelson, J. Math. Phys. 5, 332 (1964); Phys. Rev. 150, 1079 (1966). 
[3] J. G. Gilson, Proc. Camb. Phil. Soc. 64, 1061 (1968).

[4] L. S. F. Olavo, Phys. Rev. A 61, 052109 (2000).

[5] P. Blanchard et al. (eds.), Mathematical and Physical Aspects of Stochastic Mechanics (Springer, Heidelberg, 1987).

[6] K. Yasue, Int. J. Theor. Phys. 18, 861 (1979).

[7] L. De La Peña, Found. Phys. 12, 1017 (1982).

[8] A. Zeilinger, Phys. Today 52 (February), 13 (1999).

[9] W. Heisenberg, Physics and Philosophy (Harper and Row, New York, 1958), p. 129.

[10] W. Heisenberg, The Physicist's Conception of Nature, translated by A. J. Pomerans (Hartcourt Brace, New York, 1958), p. 15.

[11] S. Goldstein, Phys. Today 51 (March), 42 (1998).

[12] I. Fényes, Z. Physik 132, 81 (1951).

[13] W. Weizel, Z. Physik 134, 264 (1953); Z. Physik 135, 270 (1953); Z. Physik 136, 582 (1954).

[14] D. Kershaw, Phys. Rev. B 136, 1850 (1964).

[15] G. G. Comisar, Phys. Rev. B 138, 1332 (1965).

[16] E. Nelson, Dynamical Theories of Brownian Motion (Princeton University Press, New Jersey, 1967).

[17] E. Nelson, Quantum Fluctuations (Princeton University Press, New Jersey, 1985).

[18] F. Selleri and G. Tarozzi, Riv. Nuovo Cimento 4(2), (1981).

[19] D. Bohm and B. J. Hiley, Phys. Rep. 172, 93 (1989).

[20] L. de Broglie, C.R. Acad. Sci. (Paris) 183, 447 (1926); C.R. Acad. Sci. (Paris) 184, 273 (1927); C.R. Acad. Sci. (Paris) 185, 380 (1927); see also: The Current Interpretation of Quantum Mechanics: a Critical Study (Cambridge University Press, Cambridge, 1965).

[21] D. Bohm, Quantum Theory (Prentice Hall, Englewood Cliffs, New Jersey, 1951).

[22] D. Bohm, Phys. Rev. 85, 166 (1952); Phys. Rev. 85, 180 (1952).

[23] D. Bohm, Phys. Rev. 89, 458 (1953).

[24] D. Bohm, in: Quantum Theory, Vol 3, edited by D. R. Bates (Academic Press, New York, 1962).

[25] D. Bohm and J. P. Vigier, Phys. Rev. 96, 208 (1954).

[26] B. Brezger, L. Hackermüller, S. Uttenthaler, J. Petschinka, M. Arndt, and A. Zeilinger, Phys. Rev. Lett. 88, 100404 (2002).

[27] M. Requardt, The Underlying Substructure of Quantum Theory and the Real Nature of Quantum Phase Interference. A Natural Resolution of the Einstein-Podolski-Rosen Paradox, the Double-Slit Experiment etc. A New Stochastic-Geometric Conception of Microdynamics and Force. Unpublished manuscript, Göttingen (1981).

[28] M. J. W. Hall and M. Reginatto, J. Phys. A, Math. Gen. 35, 3289 (2002).

[29] A. Einstein, Ann. Phys. (Germany) 17, 549 (1905); Ann. Phys. (Germany) 19, 371 (1906); see also: Investigation on the Theory of the Brownian Movement, translated by A. D. Cowper (Methuen and Company, London, 1926).

[30] P. Langevin, C.R. Acad. Sci. (Paris) 146, 530 (1908).

[31] M. V. Smoluchowski, Ann. Phys. 21, 756 (1906); Ann. Phys. 48, 1103 (1915); see also: Abhandlungen über die Brownische Bewegung und verwandte Erscheinungen (Akademische Verlagsgesellschaft, Leipzig, 1923).

[32] A. D. Fokker, Ann. Phys. 43, 812 (1914).

M. Planck, Sitz. der preuß. Akademie 324 (1917).

[33] A. N. Kolmogorov, Math. Ann. 104, 415 (1931).

[34] H. Gebelein, Turbulenz (Springer, Berlin, 1935), p. 75.

[35] H. Helmholtz, Crelles J. 55, 25 (1858).

[36] Y. Aharonov and D. Bohm, Phys. Rev. 115, 485 (1959).

[37] G. Möllenstedt and W. Bayh, Phys. Bl. 18, 299 (1962).

[38] R. G. Chambers, Phys. Rev. Lett. 5, 3 (1960).

[39] B. Mielnik and G. Tengstrand, Int. J. Theor. Phys. 19, 239 (1980).

[40] J. v. Neumann, Mathematical Foundation of Quantum Mechanics, translated by R. T. Beyer (Princeton University Press, Princeton, New Jersey, 1955).

[41] J. S. Bell, Rev. Mod. Phys. 38, 447 (1966).

[42] J. S. Bell, Physics 1, 195 (1964).

[43] G. Hermann, Abhandlungen der Freis'schen Schule 6, 75 (1935).

[44] N. D. Mermin, Rev. Mod. Phys. 65, 803 (1993). 